\title{
Plasticity of Auditory Medullary-Midbrain Connectivity across Metamorphic Development in the Bullfrog, Rana catesbeiana
}

\author{
Seth S. Horowitz ${ }^{a, b}$ Judith A. Chapman ${ }^{a}$ Andrea Megela Simmons ${ }^{a, b}$ \\ ${ }^{\mathrm{a}}$ Departments of Psychology and ${ }^{\mathrm{b}}$ Neuroscience, Brown University, Providence, R.I., USA
}

\section{Key Words}

Tadpoles • Anurans • Vestibular nucleus complex •

Dorsal medullary nucleus $\cdot$ Superior olivary complex .

Torus semicircularis • Lipophilic dyes • PHA-L •

Cholera toxin $\cdot$ Metamorphosis

\begin{abstract}
On the basis of patterns of anterograde, retrograde, and bidirectional transport of tracers from both the superior olivary nucleus (SON) and the torus semicircularis (TS), we report anatomical changes in brainstem connectivity across metamorphic development in the bullfrog, Rana catesbeiana. In early and late stages of larval development (Gosner stages 25-37), anterograde or bi-directional tracers injected into the SON produce terminal/fiber label in the contralateral SON and in the ipsilateral TS. Between stages 38-41 (deaf period), only sparse or no terminal/fiber label is visible in these target nuclei. During metamorphic climax (stages 42-46), terminal/fiber label reappears in both the contralateral SON and in the ipsilateral TS, and now also in the contralateral TS. Injections of retrograde tracers into the SON fail to label cell bodies in the ipsilateral TS in deaf period animals, mirroring the previously-reported failure of retrograde transport from the TS to the ipsilateral SON during this developmental time. Bilateral cell body label emerges in the dorsal medullary nucleus and the lateral vestibular nucleus bilaterally as a result of SON transport during the late larval period, while cell body label in the contralateral TS emerges during
\end{abstract}

climax. At all larval stages, injections into the SON produce anterograde and retrograde label in the medial vestibular nucleus bilaterally. These data show anatomical stability in some pathways and plasticity in others during larval development, with the most dramatic changes occurring during

\begin{tabular}{ll}
\hline Abbreviations used in this paper \\
\hline Cer & cerebellum \\
CT $\beta$ & Vibrio cholera toxin $\beta$ subunit \\
DAP & dorsal arcuate pathway \\
DiI & $1,1^{\prime}$-dioctadecyl-3,3, $3^{\prime}, 3^{\prime}$ - -tetramethylindocarbocya- \\
& nine perchlorate \\
DMN & dorsal medullary nucleus \\
DT & dorsal tegmentum \\
HRP & horseradish peroxidase \\
LLa & anterior lateral line nucleus \\
LLnp & lateral line neuropil \\
LVN & lateral vestibular nucleus \\
mr & medial reticular zone \\
MVN & medial vestibular nucleus \\
OT & optic tectum \\
OV & optic ventricle \\
PHA-L & Phaseolus vulgaris leucoagglutinin \\
SON & superior olivary nucleus \\
TS & torus semicircularis \\
TSl & laminar nucleus of the torus semicircularis \\
VAP & ventral arcuate pathway \\
VII & seventh motor nucleus \\
VT & ventral tegmentum \\
\end{tabular}

\section{KARGER}

() 2007 S. Karger AG, Basel

Fax +41613061234

E-Mail karger@karger.ch

www.karger.com www.karger.com/bbe
Andrea M. Simmons

Department of Psychology

Box 1853, Brown University

Providence, RI 02912 (USA)

Tel. +1 401863 2283, Fax +1 401863 1300,E-Mail Andrea_Simmons@brown.edu 
the deaf period and metamorphic climax. Animals in metamorphic climax show patterns of connectivity similar to that of froglets and adults, indicating the maturation during climax of central anatomical substrates for hearing in air.

Copyright @ 2007 S. Karger AG, Basel

\section{Introduction}

The central nervous system of anuran amphibians undergoes substantial reorganization across metamorphic development. This reorganization affects all sensory, motor, and vegetative systems to different degrees and at different time courses [Spaeti, 1978], and is particularly pronounced in those anurans, such as the bullfrog (Rana catesbeiana), that adopt partly terrestrial lifestyles after metamorphosis. The drastic change in sensory environment from totally aquatic to partly terrestrial imposes particular constraints on the type of auditory input available to the animal. These constraints can be expected to drive anatomical and functional changes in the auditory system during the metamorphic shift from tadpole to frog.

The bullfrog's brain as a whole increases in size over metamorphic development, and this change in size is accompanied by changes in the organization of central auditory nuclei (fig. 1). This is particularly obvious in an examination of the dorsal medullary nucleus (DMN), the medullary termination site of projections from the auditory endorgans [Jacoby and Rubinson, 1983; Fritzsch et al., 1984]. In early larval stages, the DMN is located laterally in the medulla, near the entry point of the eighth cranial nerve. It is bounded medially by the lateral line neuropil and dorsally by what we are terming the medial vestibular nucleus (MVN; fig. 1A). By the end of metamorphic climax and into the froglet period, the DMN has not only increased in overall size and cell number [Chapman et al., 2006], but it has also migrated to the more medial and dorsal location seen in froglets and adults [Jacoby and Rubinson, 1983; Fritzsch et al., 1984; Kumaresan et al., 1998; fig. 1B]. In Rana and other frogs that do not remain totally aquatic after metamorphosis, this change in position occurs concomitantly with the degeneration of the lateral line neuropil and lateral line nuclei [Larsell, 1934; Fritzsch et al., 1984, 1988]. In contrast, the superior olivary nucleus (SON) does not appear to migrate in position but it does vary considerably in cell density and nuclear volume over larval development [Templin and Simmons, 2005]. Three major subdivisions of the midbrain torus semicircularis (TS) that are apparent in adult frogs [Feng and Lin, 1991] are largely undifferen-

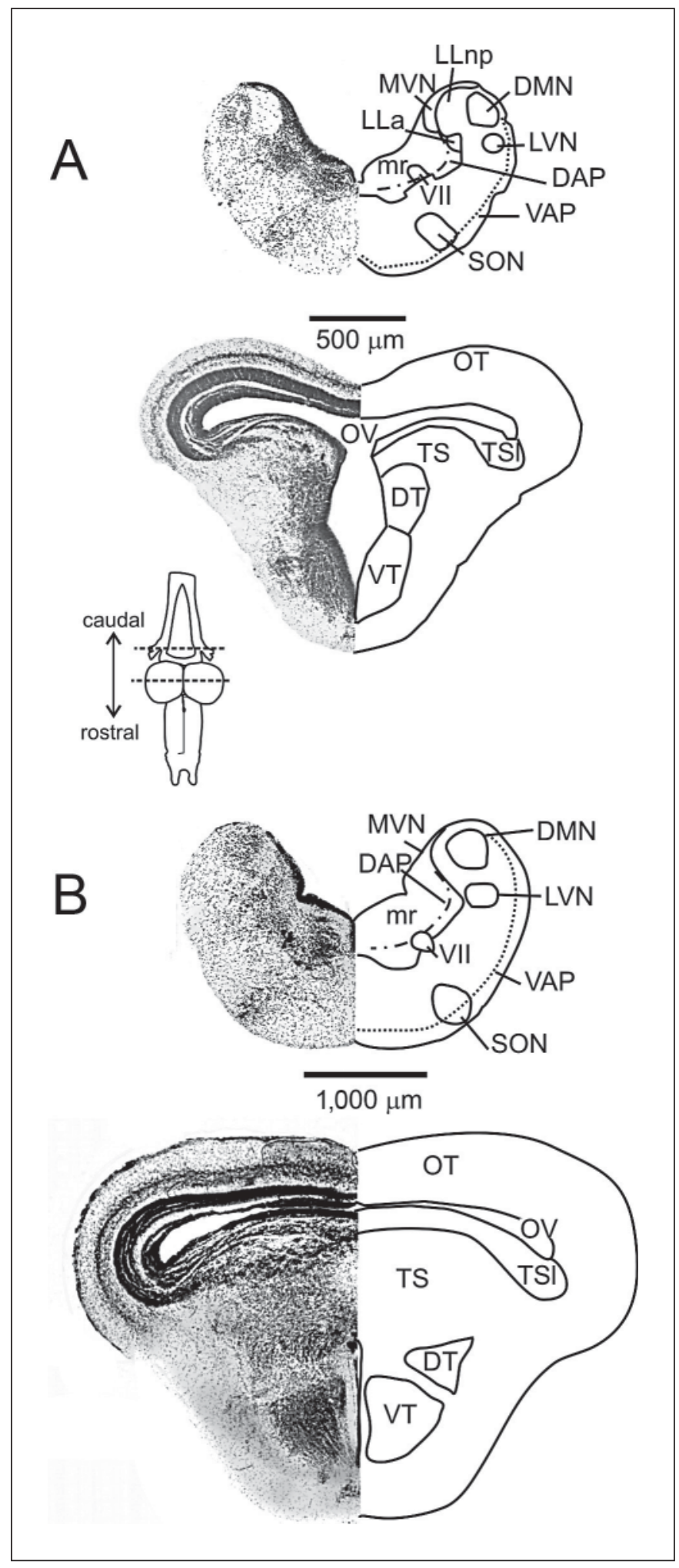

Fig. 1. Diagrams of representative brain sections from a stage 30 tadpole $(\mathbf{A})$ and a froglet $(\mathbf{B})$. Sections were taken at the approximate levels indicated on the dorsal view of the brain (left). Scale bars $\mathbf{A}=500 \mu \mathrm{m} ; \mathbf{B}=1,000 \mu \mathrm{m}$. For abbreviations, see list. 
tiated in tadpoles, and it is not until metamorphic climax and the froglet period that these divisions become morphologically distinct [Boatright-Horowitz and Simmons, 1995; Kumaresan et al., 1998].

Neural responses to pressure stimulation can be recorded from the TS of bullfrog tadpoles at all stages of metamorphic development, but the spectral and temporal sensitivity of these responses vary considerably. Pure tone audiograms recorded from the TS of tadpoles in early and late larval stages exhibit a high-pass shape, with lowest thresholds around 2,000 Hz. Responses to complex sounds show a broad frequency range of phaselocked activity (up to amplitude modulation rates as high as $200-250 \mathrm{~Hz}$ ). In contrast, audiograms of tadpoles in metamorphic climax stages are V- or U-shaped, with most sensitive frequencies around $1,000-1,500 \mathrm{~Hz}$ and greater sensitivity to low frequency tones than seen in younger animals [Boatright-Horowitz and Simmons, 1997]. The frequency range of phase-locked activity is also more restricted [Boatright-Horowitz et al., 1999]. For a limited developmental period before the onset of metamorphic climax, no or extremely limited auditory responsiveness can be detected in the TS. During this same developmental time span, there is also a transient loss of afferent connectivity to the TS from the SON, as shown by failure of retrograde transport of tracer from the TS. Transport is again visible during metamorphic climax. This transient period (Gosner stages 38-41) has been termed the deaf period [Boatright-Horowitz and Simmons, 1997] on the basis of these changes in physiological responsiveness and anatomical connectivity.

The purpose of this study is to more fully examine changes in brainstem connectivity across metamorphic development. The anatomical data of Boatright-Horowitz and Simmons [1997] were based solely on the retrograde transport of tracer from TS injections. Anterograde patterns of connectivity were not examined, nor was connectivity of the TS or of the SON to other medullary nuclei fully described. Here, we present data based on multiple tract tracing techniques, with anterograde, retrograde and bi-directional tracers, to examine changes in both afferent and efferent connectivity of auditory brainstem nuclei across the entire span of postembryonic development, from early larval tadpoles to adults. We show here that the disruption of afferent connectivity from the SON to the TS during the deaf period is mirrored by a disruption of efferent connectivity from the TS to the SON during this same time span. We also describe several aspects of brainstem circuitry, found in adult frogs, some of which first emerge during the deaf period where- as others emerge only during metamorphic climax, in final preparation for the transition from a fully aquatic to an amphibious lifestyle.

\section{Materials and Methods}

\section{Animals}

The original research reported herein was performed under guidelines established by Brown University Institutional Animal Care and Use Committee, and consistent with federal regulations. Rana catesbeiana tadpoles and adults were purchased from commercial suppliers (Sullivan, Tenn., USA; Dozier Lester, L.A., USA). Only free-swimming postembryonic tadpoles were used for experiments. They were staged by external morphological criteria based on Gosner [1960] and broadly categorized into four groups [McDiarmid and Altig, 1999]: early larval stages (stages $25-30, \mathrm{n}=15$, undeveloped hindlimb buds); late larval stages (stages $31-37, \mathrm{n}=15$, progressive differentiation of hindlimbs); deaf period (stages $38-41, \mathrm{n}=22$, final hindlimb differentiation and internal forelimb development) and metamorphic climax (stages $42-46, \mathrm{n}=22$, fully-developed hindlimbs, emergence and full development of forelimbs, tail resorption, head and mouth transformation). Postmetamorphic animals included froglets ( $\mathrm{n}=6$, snout-vent length, SVL $\leq 5.5 \mathrm{~cm})$ and adults $(\mathrm{n}=9$, SVL $>10 \mathrm{~cm}$ ). Tadpoles were housed in plastic aquaria containing dechlorinated aerated water and were fed cooked unsalted spinach. Froglets and adults were housed in plastic terraria containing both soil and water and were fed live crickets. The colony room was maintained at temperatures ranging from $25-28^{\circ} \mathrm{C}$ and on a 12:12 light:dark cycle.

\section{Tracer Deposits}

In 20 animals, Phaseolus vulgaris leucoagglutinin [PHA-L, L32455, a primarily anterograde tracer; Gerfen and Sawchenko, 1984] conjugated to AlexaFluor 568 was co-injected with Vibrio cholera toxin $\beta$ subunit [CT $\beta$, C22841, a primarily retrograde tracer; Luppi et al., 1990] conjugated to AlexaFluor 488 (both from Molecular Probes/Invitrogen, Eugene OR) into either the TS or the SON. The simultaneous use of an anterograde and a retrograde tracer allowed us to investigate the distribution of both afferent and efferent processes in the same brains.

In 31 animals, the tracers used for pressure injections were lipophilic carbocyanine dyes: 1,1'-dioctadecyl-5,5'-diphenyl$3,3,3^{\prime}, 3^{\prime}$-tetramethylindocarbocyanine chloride (DiI-Ph, D7779); or $1,1^{\prime}$-dioctadecyl-3,3,3',3'- tetramethylindocarbocyanine perchlorate (DiI, D282), both from Molecular Probes/Invitrogen. Lipophilic carbocyanine dyes are transported in the neuronal lipid bilayer and travel bidirectionally [Godement et al., 1987]. DiI and DiI-Ph absorb and emit at similar wavelengths and their neuronal travel characteristics are indistinguishable. However, DiI-Ph has attached phenyl constituents resulting in greater fluorescence intensity than DiI. Because both DiI and DiI-Ph have similar bidirectional propagation characteristics, data from both tracers will be presented jointly. Lipophilic dye injection was made in fixed tissue in 18 animals and in live tissue in 13 animals. Tracer transport speed in fixed tissue is typically $0.2-0.6 \mathrm{~mm}$ /day and up to $6 \mathrm{~mm} /$ day in living tissue [Godement et al., 1987]. We adjusted the time interval between tracer deposition and tissue slicing for 
transport in live and dead tissue. For animals in which DiI was microinjected into fixed brain tissue, transport time was approximately $30-40$ days at $4^{\circ} \mathrm{C}$.

In 9 animals, HRP was iontophoresed into the TS, following procedures described previously [Boatright-Horowitz and Simmons, 1997].

Animals were anesthetized for surgery by immersion in $0.15 \%$ (tadpoles and froglets) or $0.6 \%$ (adults) tricaine methanesulfonate (MS 222, pH 8.0; Sigma) until all reflexes disappeared. They were wrapped in wet gauze and the brainstem was exposed by removal of overlying cartilaginous tissue (tadpoles) or bone drilling (froglets and adults). For SON microinjection sites, the lymphatic tissue and blood vessels overlying the medulla were gently retracted in order to minimize blood loss to the animal. The eighth nerve was located to aid in the identification of the approximate rostral-caudal position of the SON. For pressure injections, tracer was backfilled into a glass micropipette (20-40 $\mu \mathrm{m}$ tip diameter). The pipette was held by a micromanipulator (Narishige, East Meadow, N.Y., USA) attached to a manual microinjector (Sutter Instruments, Novato, Calif., USA) and gently placed into the appropriate brain nucleus using coordinates and depths based on an atlas developed in our laboratory. A criterion amount of $0.20-0.25 \mu \mathrm{l}$ of tracer was microinjected into each brain. The micropipette remained in place for $5 \mathrm{~min}$ after microinjection to allow the tracer to permeate the brain region. The site was then thoroughly rinsed with $0.9 \%$ sterile saline, and antiseptic Gelfoam (Upjohn, Kalamazoo, Mich., USA) was placed in the wound. The skin was closed using 5/0 Vicryl (Ethicon, Somerville, N.J., USA) sutures and triple antibiotic containing lidocaine (Neosporin; Pfizer, Cambridge, Mass., USA) was topically applied. Animals were allowed to recover for 2 (CTß/PHA-L) or 3 days (lipophilic dyes, HRP).

Following these recovery times, the animals were terminally anesthetized by immersion in $0.6 \%$ MS 222 until all reflexes disappeared, then transcardially perfused with heparinated $0.9 \%$ saline followed by $4 \%$ paraformaldehyde ( $\mathrm{pH} 7.4$ ). Brains were removed and postfixed overnight at $4^{\circ} \mathrm{C}$ in $4 \%$ paraformaldehyde. Overlying meninges were removed and brains were embedded in agarose ( $5 \%$ in saline) and sliced by vibratomy ( $50 \mu \mathrm{m}$ coronal or $75 \mu \mathrm{m}$ sagittal sections). For experiments using CT $\beta /$ PHAL or lipophilic dyes, two sets of alternating brain sections were placed on gelatin-subbed slides. One set was directly mounted using AquaMount (Polysciences, Warrington, Pa., USA) for visualization of fluorescence. The second set was placed in $0.5 \%(\mathrm{w} / \mathrm{v}) \mathrm{cre}$ syl violet acetate staining solution $(\mathrm{pH}$ to 3.7 using glacial acetic acid) for 10 min, washed in $\mathrm{H}_{2} \mathrm{O}$, and dehydrated using a graded ethanol series. This procedure was followed by removal of background staining with Citrisolv (Fisher Scientific, Pittsburgh, Pa., USA) and a $30 \mathrm{sec}$ wash in xylenes (Fisher Scientific). Sections were mounted using Cytoseal acrylic mounting medium (VWR, Bridgeport, N.J., USA). For experiments using HRP, all sections were processed for reaction product (brown/black) using the Hanker-Yates method [Hanker et al., 1977] and then counterstained with cresyl violet.

\section{Image Processing and Analyses}

In order to quantify nuclear boundaries in larval tissue, we generated anatomical atlases derived from cresyl violet-stained brain sections from 40 animals that spanned the range of postembryonic development. Tissue for these atlases were sliced either coronally $(50 \mu \mathrm{m})$ or sagittally $(75 \mu \mathrm{m})$. Stained sections were im- aged, combined and brightness/contrast balanced into single images using photomosaic software (Photoshop CS, Adobe) and saved as 24 bit RGB TIFF files. The blue channel of the mosaic images was then low-pass filtered to combine individual cells into nuclear clusters, a contour filter applied to create boundary edges, and the edges smoothed using vector graphics drawing software (Corel Draw v. 11, Corel). These images were then saved as 1 bit (b/w) GIF files to create line drawings that comprised the atlas. Atlas figures were saved with transparent backgrounds and overlayed on bitmapped images to facilitate localization of cell bodies, terminals and fibers within the nuclear boundaries.

To identify injection sites and patterns of transport, sections were visualized and imaged using an Olympus BX60 research microscope (Melville, N.Y., USA) and Optronics digital camera (Goleta, Calif., USA) equipped with custom-made fluorescence cubes. Images were acquired using a Dell Pentium IV 2.4GHz computer running MagnaFire image acquisition and analysis software (Optronics, Goleta, Calif., USA) and were stored as 24-bit RGB TIFF images. Images were contrast adjusted and color balanced to match luminance between sections. Custom wavelet deconvolution filters (Registax) were applied to fluorescent images to highlight fine structures. Schematization of cell bodies and nerve terminal location was carried out by dividing 24 bit RGB TIFF files into red, green and blue 8 bit grayscale images, converting these to thresholded binary images, tracing the edges of the objects and passing the tracings through particle size filters with parameters based on a selection of 25 randomly chosen cells from the regions of interest (Image J, NIH).

The amount of cell body and fiber label was categorized as none (no label in any section), sparse (inconsistent label, with 0 or 1 occasional cell body or fiber per 50 or $75 \mu \mathrm{m}$ section; $-/+$ in tables 1,2$)$, light $(1-2$ cell bodies or fibers; + in tables 1,2$)$, modest ( $3-4$ cell bodies/fibers; ++ in tables 1,2$)$, moderate $(5-8$ cell bodies/fibers; +++ in tables 1,2$)$ and heavy ( 9 or more cell bodies/fibers per section; ++++ in tables 1,2 ). The amount of terminal (puncta) label was categorized similarly, but with the number of cell bodies around which clusters of terminals were found as the unit of measure. The use of an individual tissue section as the unit of categorization allowed us to directly compare label across developmental stages, despite the differences in numbers of sections through the brainstem across development [Templin and Simmons, 2005; Chapman et al., 2006; Simmons et al., 2006]. Categorizing the amount of label was made by all 3 authors independently, and the tables reflect the most common result from all sections in particular developmental groups.

\section{Anatomical Boundaries and Terminology}

Cresyl violet-stained sections and schematics of the medulla and midbrain at two developmental stages (early larval tadpole and froglet) are shown in figure 1 . Nuclear boundaries in the tadpole and froglet brains are based on our own in-house atlases, supplemented by relevant literature from other laboratories [Jacoby and Rubinson, 1983, 1984; Fritzsch et al., 1984]. Boundaries in the adult brain are based on Matesz [1979], Wilczynski [1981], Montgomery [1988], Will and Fritzsch [1988], Feng and Lin [1991], McCormick [1999] and Matesz et al. [2002]. The DMN has been variously called the nucleus cochlearis [Larsell, 1934; Matesz, 1979], the acoustic nucleus [Jacoby and Rubinson, 1983] and the dorsal lateral nucleus [Will and Fritzsch, 1988; Chapman et al., 2006]. What we are identifying as the anterior lateral line nucleus 
Table 1. Qualitative label in target nuclei following tracer injections in the SON

\begin{tabular}{|c|c|c|c|c|c|c|}
\hline \multirow{2}{*}{$\begin{array}{l}\text { Target nuclei } \\
\text { Early (25-30) }\end{array}$} & \multicolumn{2}{|c|}{$\begin{array}{l}\text { Cell bodies } \\
\text { (c/i) }\end{array}$} & \multicolumn{2}{|c|}{$\begin{array}{l}\text { Terminals } \\
(\mathrm{c} / \mathrm{i})\end{array}$} & \multicolumn{2}{|c|}{$\begin{array}{l}\text { Fibers } \\
(\mathrm{c} / \mathrm{i})\end{array}$} \\
\hline & & & & & & \\
\hline DMN & ++ & - & + & $-1+$ & + & $-1+$ \\
\hline SON & ++ & INJ & + & INJ & ++ & INJ \\
\hline MVN & + & + & + & + & + & + \\
\hline LVN & - & - & - & - & - & - \\
\hline LLa & ++ & ++ & - & - & + & + \\
\hline TS & - & $-1+$ & - & ++ & + & + \\
\hline \multicolumn{7}{|l|}{ Late $(31-37)$} \\
\hline DMN & ++ & $-1+$ & + & $-1+$ & + & $-1+$ \\
\hline SON & ++ & INJ & + & INJ & + & INJ \\
\hline MVN & + & + & + & + & + & ++ \\
\hline LVN & + & + & $-1+$ & $-1+$ & $-1+$ & - \\
\hline LLa & ++ & ++ & - & - & + & + \\
\hline TS & - & + & - & ++ & + & + \\
\hline \multicolumn{7}{|l|}{ Deaf (38-41) } \\
\hline $\mathrm{DMN}$ & + & $-1+$ & $-1+$ & $-1+$ & - & $-1+$ \\
\hline SON & $-1+$ & INJ & $-1+$ & INJ & - & INJ \\
\hline MVN & + & + & $-1+$ & $-1+$ & + & + \\
\hline LVN & + & + & $-1+$ & $-1+$ & - & + \\
\hline LLa & - & $-1+$ & - & - & - & - \\
\hline TS & - & - & - & - & - & - \\
\hline \multicolumn{7}{|c|}{ Climax (42-46) } \\
\hline $\mathrm{DMN}$ & ++ & + & ++ & ++ & ++ & ++ \\
\hline SON & ++ & INJ & ++ & INJ & ++ & INJ \\
\hline MVN & ++ & ++ & + & + & + & ++ \\
\hline LVN & ++ & ++ & + & + & + & + \\
\hline LLa & - & - & - & - & $-1+$ & - \\
\hline TS & ++ & ++ & + & ++ & + & ++ \\
\hline \multicolumn{7}{|l|}{ Froglet } \\
\hline DMN & ++ & + & ++ & ++ & ++ & ++ \\
\hline SON & ++ & INJ & ++ & INJ & ++ & INJ \\
\hline MVN & ++ & ++ & + & + & + & + \\
\hline LVN & ++ & ++ & + & + & + & + \\
\hline TS & ++ & ++ & + & ++ & + & ++ \\
\hline
\end{tabular}

Data are presented separately for different developmental stage groups. $\mathrm{c}=$ Contralateral; $\mathrm{i}=$ ipsilateral. Label is categorized as - none; -/+ sparse; + light; ++ modest; +++ moderate; ++++ heavy. INJ indicates injection site. Entries based on the most representative data from each developmental group, labeled with different tracers.
Table 2. Qualitative label in target nuclei following tracer injections in the TS

\begin{tabular}{|c|c|c|c|c|c|c|}
\hline \multirow{2}{*}{$\begin{array}{l}\text { Target nuclei } \\
\text { Early (25-30) }\end{array}$} & \multicolumn{2}{|c|}{$\begin{array}{l}\text { Cell bodies } \\
(\mathrm{c} / \mathrm{i})\end{array}$} & \multicolumn{2}{|c|}{$\begin{array}{l}\text { Terminals } \\
(\mathrm{c} / \mathrm{i})\end{array}$} & \multicolumn{2}{|c|}{$\begin{array}{l}\text { Fibers } \\
(\mathrm{c} / \mathrm{i})\end{array}$} \\
\hline & & & & & & \\
\hline DMN & + & $-1+$ & + & - & + & - \\
\hline SON & - & ++ & - & ++ & ++ & ++ \\
\hline MVN & + & + & + & + & $-1+$ & $-1+$ \\
\hline LVN & $-1+$ & + & - & - & - & - \\
\hline LLa & $-1+$ & + & - & - & $-1+$ & $-1+$ \\
\hline TS & ++ & INJ & + & INJ & + & INJ \\
\hline \multicolumn{7}{|l|}{ Late $(31-37)$} \\
\hline DMN & ++ & + & ++ & $-1+$ & + & + \\
\hline SON & - & ++ & - & ++ & ++ & ++ \\
\hline MVN & + & + & + & + & + & + \\
\hline LVN & + & + & - & - & - & + \\
\hline LLa & + & + & - & - & $-1+$ & $-1+$ \\
\hline TS & ++ & INJ & + & INJ & + & INJ \\
\hline \multicolumn{7}{|l|}{ Deaf (38-41) } \\
\hline DMN & $-1+$ & - & + & $-1+$ & + & $-1+$ \\
\hline SON & - & - & - & $-1+$ & $-1+$ & - \\
\hline MVN & + & $-1+$ & + & + & ++ & ++ \\
\hline LVN & + & + & - & - & $-1+$ & $-1+$ \\
\hline LLa & - & $-1+$ & - & - & - & - \\
\hline TS & + & INJ & - & INJ & + & INJ \\
\hline \multicolumn{7}{|c|}{ Climax (42-46) } \\
\hline DMN & ++ & + & ++ & + & + & $-1+$ \\
\hline SON & + & ++ & + & ++ & + & ++ \\
\hline MVN & ++ & ++ & + & + & + & + \\
\hline LVN & ++ & ++ & - & - & - & - \\
\hline LLa & - & - & - & - & - & - \\
\hline TS & +++ & INJ & ++ & INJ & + & INJ \\
\hline
\end{tabular}

\begin{tabular}{lllllll}
\hline Froglet & & & & & & \\
DMN & ++ & + & ++ & + & ++ & + \\
SON & + & ++ & + & ++ & + & + \\
MVN & + & ++ & + & + & + & +++ \\
LVN & ++ & ++ & - & - & + & $-/+$ \\
TS & +++ & INJ & ++ & INJ & ++ & INJ \\
\hline Adult & & & & & & \\
DMN & ++ & + & ++ & + & +++ & ++ \\
SON & + & +++ & ++ & ++ & ++ & ++++ \\
MVN & ++ & ++ & + & + & ++ & +++ \\
LVN & ++ & ++ & - & - & + & $-/+$ \\
TS & ++++ & INJ & ++ & INJ & +++ & INJ \\
\hline
\end{tabular}

Data are presented separately for different developmental stage groups. $\mathrm{c}=$ Contralateral; $\mathrm{i}=$ ipsilateral. Label is categorized as in table 1. INJ indicates injection site. Entries based on the most representative data from each developmental group, labeled with different tracers. 
(LLa) is the rostral and ventral part of what Fritzsch et al. [1984] termed the intermediate nucleus. According to those authors, this particular part of the intermediate nucleus degenerates during metamorphic climax, whereas the more caudal and dorsal part of the intermediate nucleus forms the caudal nucleus (nucleus caudalis) as earlier described by Opdam et al. [1976]. We do not distinguish, in our material, between a caudal nucleus and the medial vestibular nucleus (MVN). Instead, we use the term MVN to be consistent with the terminology used in adult Rana by Montgomery [1988] and Matesz et al. [2002]; these authors do not differentiate a separate caudal nucleus. The LVN as identified in this study has been termed the vestibular nucleus, Deiters nucleus or the ventral nucleus of the eighth nerve [Matesz, 1979; Fritzsch et al. 1984; Montgomery, 1988]; its dorsal area also includes what Matesz [1979] termed the saccular nucleus. We do not distinguish a separate saccular nucleus in our material. In larval Rana, there are no published data showing distribution of afferents from all individual endorgans in the otic capsule to medullary termination sites; such data are necessary to differentiate a saccular nucleus from the DMN and LVN. We also do not differentiate a separate descending vestibular nucleus; even in adult Rana, the distinction between this nucleus and the MVN and LVN can be difficult to ascertain [Reichenberger et al., 1997].

The TS in adult Rana is divided into three main subdivisions of laminar, principal, and magnocellular nuclei [Feng and Lin, 1991]. In the tadpole brain, these subdivisions cannot be easily identified because of differences in cell density and patterns of lamination across development. For example, the area described in adults as the principal nucleus does not attain its distinctive pattern of lamination until metamorphic climax stages [Simmons and Chapman, 2002]. Moreover, until metamorphic climax stages, the optic ventricle and aqueduct of Sylvius are continuous from the level of the nucleus isthmus up to the level of the oculomotor nucleus $[\mathrm{Ku}$ maresan et al., 1998; fig. 1A]. We refer to the area of layered cells immediately ventral to the optic ventricle as the developing laminar nucleus (TSl; described as ventricular TS by Boatright-Horowitz and Simmons [1997]), and we do not distinguish a principal or magnocellular nucleus in any but adult brains.

\section{Results}

\section{Injection Sites}

Injections into the SON were performed in 26 animals of PHA-L/CT $\beta(n=10)$ or DiI/DiI-Ph $(n=16)$. Injections into the TS were made in 34 animals using HRP $(n=9)$, PHA-L/CT $\beta(n=10)$ or DiI/DiI-Ph $(n=15)$. These later results are compared with data previously reported by Boatright-Horowitz and Simmons [1997] in a separate sample of 29 larval animals in which HRP was iontophoresed into the TS. Not every site received all combinations of tracers. Cell body label was apparent after both HRP and CT $\beta$ injections, and often visible, but less so, after DiI injections. We did not observe any retrograde cell body label after PHA-L injections [Antal and Petko, 1990]. Terminal label was visible after both PHA-L and HRP injections and sometimes with DiI. DiI and HRP produced the most fiber label, although segments of fibers were often labeled with PHA-L/CT $\beta$. Afferent and efferent patterns of transport from these injections, based on the most consistent results seen in each developmental group, are presented schematically in figure 2 , and in tabular form in table 1 (SON injections) and 2 (TS injections). Data in the tables include only those results that are most representative of and consistent across the different tracers (the pattern of results from individual brains may vary depending on the size of the injection site; this kind of variability is noted in the text). Data from those injections in which the injection site could not be located are not included in the tables $(n=14$ additional animals). Data from those animals in which the injection site was not constrained within the boundaries of the target nucleus or was centered within a non-auditory area $(n=7)$ were used for comparative purposes. Below, we first describe our results from SON injections, then from TS injections, organized by stage group. To facilitate descriptions of the results, data from early and late larval stages are presented together, and those from the other stage groups are presented separately.

\section{Early (25-30) and Late (31-37) Larval Stages}

Retrograde and anterograde transport patterns after tracer deposits in the SON and TS in early larval (fig. 2A, F) and late larval (figs. 2B, G) stages are generally consistent across these two developmental stage groups, with some exceptions noted below.

SON Injections. In both early and late larval brains, retrograde tracer injections into the SON produced modest cell body label in the contralateral SON (cSON; fig. 3E) and in the contralateral DMN (cDMN). There was light to modest label bilaterally in the LLa and MVN. In late larval animals, cell body label was visible in the ipsilateral DMN (iDMN; fig. 3A), the ipsilateral LVN (iLVN), and in the contralateral LVN (cLVN; fig. 3C). In early larval stages, label in these three nuclei was absent. Large injections typically labeled cells in the VII motor nucleus bilaterally. Light to modest cell body label, depending on the size of the injection, was observed in the iTS, particularly in lateral areas (fig. 3G); however, there was no cell body label in the medial iTS, or in any part of the cTS, even after large deposits of tracer.

Anterograde PHA-L or bi-directional DiI injections in the SON yielded light terminal label in the cSON, particularly around its dorsal margin, and in the cDMN, again around the dorsal margin. Light terminal label was noted in the MVN bilaterally. There was sparse terminal label in the iDMN, but no terminal label in the LLa bilat- 
erally. In late larval stages, sparse terminal label appeared in the LVN bilaterally. The lateral regions of the iTS showed light to modest terminal label, depending on size of the injection site. Large injections produced some label extending more ventrally, dorsal and lateral to the ventral tegmental region. However, even with large injections, no terminal label could be seen in either the medial regions of the iTS, or in any region of the cTS.

Bidirectional label following DiI injections in the SON produced modest fiber label around the cSON and in fibers extending primarily along the ventral arcuate pathway (VAP). The dorsal arcuate pathway (DAP) showed more limited, light label, particularly in the medial reticular region. Light fiber label was observed around the cDMN, cLVN, and cMVN, with modest label observed in the region of the iMVN and in ascending fibers of the lateral lemniscus. Only sparse fiber label around the iDMN was observed, while light fiber label was seen in the lateral regions of both the cTS and the iTS.

TS Injections. In both early and late larval animals, CT $\beta$ injections into the lateral (fig. 2F) or medial (fig. 2G) TS consistently yielded light to modest, depending on size of the injection site, cell label in the cDMN (fig. 4A) and bilaterally in the MVN (fig. 4C). Light to modest cell body label was also observed in the ipsilateral SON (iSON). In 3 animals in these stage groups given CT $\beta$ / PHA-L co-injections, 1-2 scattered cells in the iSON labeled with CT $\beta$ were surrounded by PHA-L labeled terminal fields (fig. 4D). Such a pattern of label indicates a reciprocal connection between the TS and the SON. Sparse to light cell body label was observed in the cLVN, iLVN, contralateral LLa, and ipsilateral Lla. Large injections labeled modest to moderate numbers of cells throughout the reticular gray, particularly in the medial reticular zone, and occasionally labeled cells in the VII motor nucleus. Even these large injections did not label cell bodies in the cSON, however. Within the midbrain, TS injections labeled modest numbers of cells throughout the rostral-caudal extent of the iTS. There was modest cell body label in the cTS, extending in and around the ventral border of the developing TSl (fig. 4E). Large injections also labeled cells near or around the contralateral tegmental nuclei.

A difference between results in early and late larval stages is seen in the label of the iDMN after TS injections (table 2). In early larval stages, only sparse, inconsistent label in the iDMN was observed, even after large tracer deposits. In contrast, injections of CT $\beta$, particularly in the medial TS, of late larval animals consistently yielded light cell body label in the iDMN.

Metamorphic Changes in Auditory Connectivity
Anterograde TS projections labeled by PHA-L from the same TS injection site yielded light terminal label in the MVN bilaterally, light to modest terminal label in the cDMN and modest terminal label in the iSON. In late larval stages, light to modest terminal label was observed in the iDMN, while in early larval stages, only sparse terminal label was observed in this nucleus. In neither stage group was terminal label in the cSON observed. Light terminal label was also observed in the cTS ventral to the area of cell body label in the TSl.

DiI injections in the TS yielded light to modest fiber label in the DAP, with sparse to light fiber label, depending on size of the injection site, terminating near the cDMN, and sparse fiber label overlapping the LLa and MVN bilaterally. The VAP was more heavily labeled. No fiber label was observed terminating near the iDMN or the LVN bilaterally. Light fiber label was also observed in the lateral region of the cTS.

\section{Deaf Period Stages (38-41)}

The patterns of afferent and efferent connectivity seen in deaf period animals differed considerably from those observed in both early and late larval animals. Very limited transport resulted from either SON (fig. 3B, D, F, H; $5 \mathrm{~A}$ ) or TS injections (fig. 5B).

SON Injections. Injections of CT $\beta$ into the SON produced light cell body label in the cDMN, compared to the modest label consistently seen in early and late stage larval animals. Sparse cell body label was seen in the iDMN, again consistent with the results in younger animals (fig. 3B). There was only sparse cell body label in the cSON (fig. 3F), compared to the modest label seen in early and late larval animals. Label of the cLVN was light to modest, depending on the size of the injection site. LLa label was sparse or absent both ipsilaterally and contralaterally. No cell body label could be visualized within the boundaries of the TS, either ipsilaterally or contralaterally. In one animal, sparse cell body label in the iTS was seen after a very large injection. On the other hand, transport was still visible to the MVN bilaterally (fig. 3D), even after small injections.

PHA-L and DiI anterograde label following SON injection was also highly constrained, showing no or only sparse terminal label in the DMN and MVN bilaterally and in the cSON (fig. 3B,D,F). Limited terminal label was observed in and around the medial reticular zone. Sparse terminal label was observed in the cLVN. Compared to the modest terminal label of the iTS in early and late stage animals, there was no terminal label in the iTS of deaf period animals. Consistent with the findings in early and 

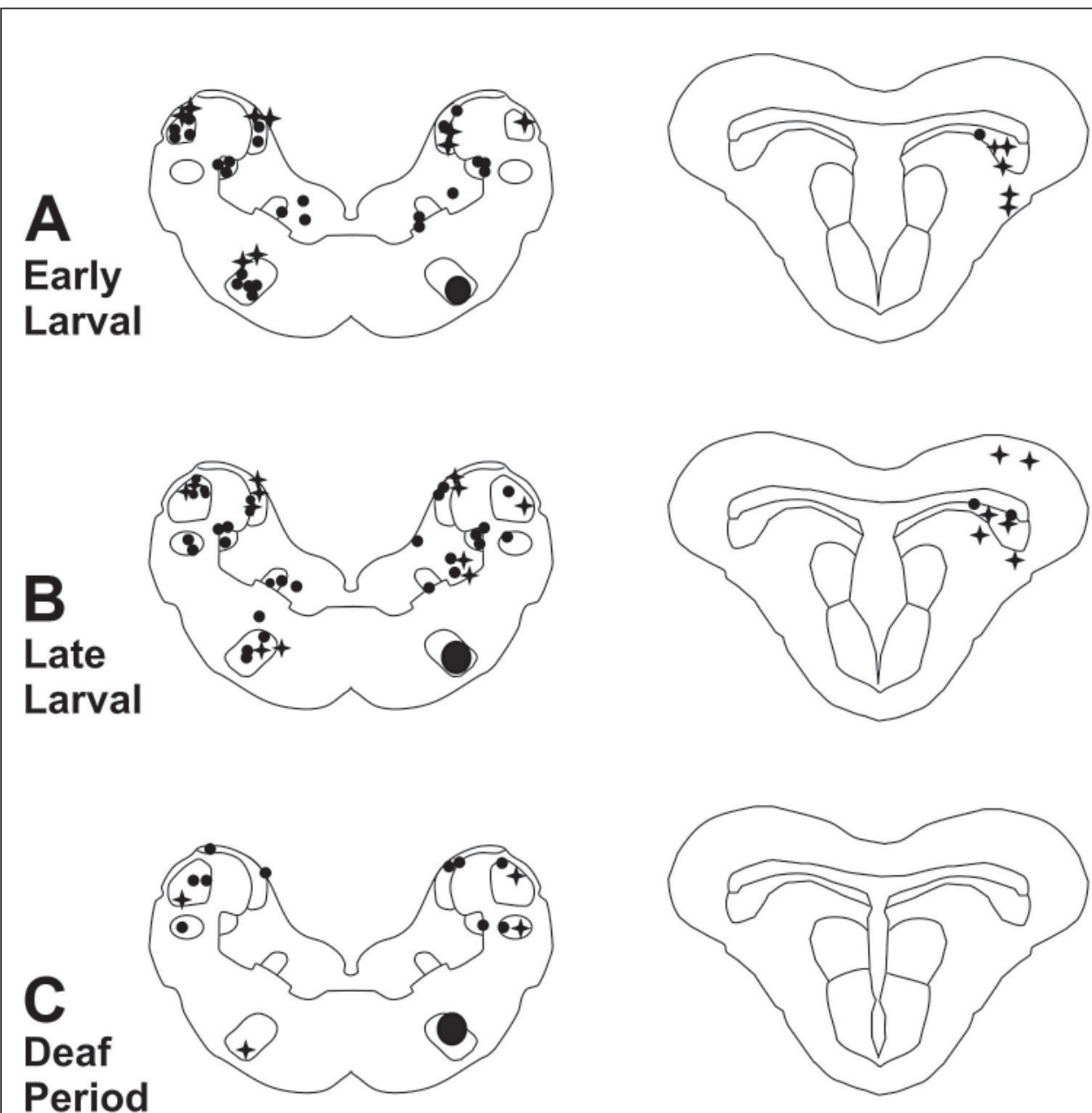

Fig. 2. Distribution of labeled cell bodies and terminals following tracer injections in the superior olivary nucleus (A-E) for medulla and midbrain in early larval (A), late larval (B), deaf period (C), metamorphic climax (D), and postmetamorphic froglets (E). Injections in the torus semicircularis (F-K) for medulla and midbrain in early larval (F), late larval (G), deaf period (H), metamorphic climax (I), froglets (J), and adults (K). cell bodies, $\bullet$ nerve terminals. Figures are schematic only (not drawn to scale) and are based on sections from representative animals in each stage group. All injection sites have been normalized to the right side for ease of comparison. Large black oval represents approximate site and extent of tracer injection. Fibers omitted for clarity.
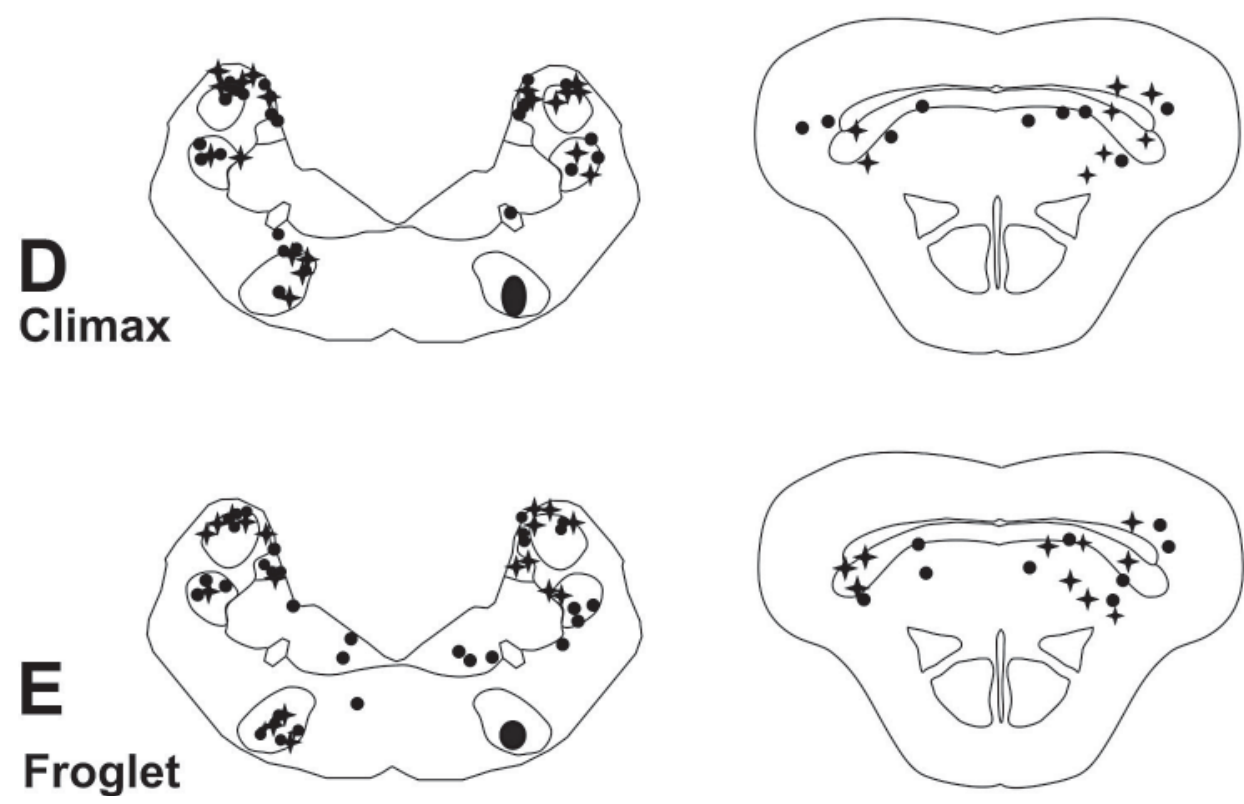


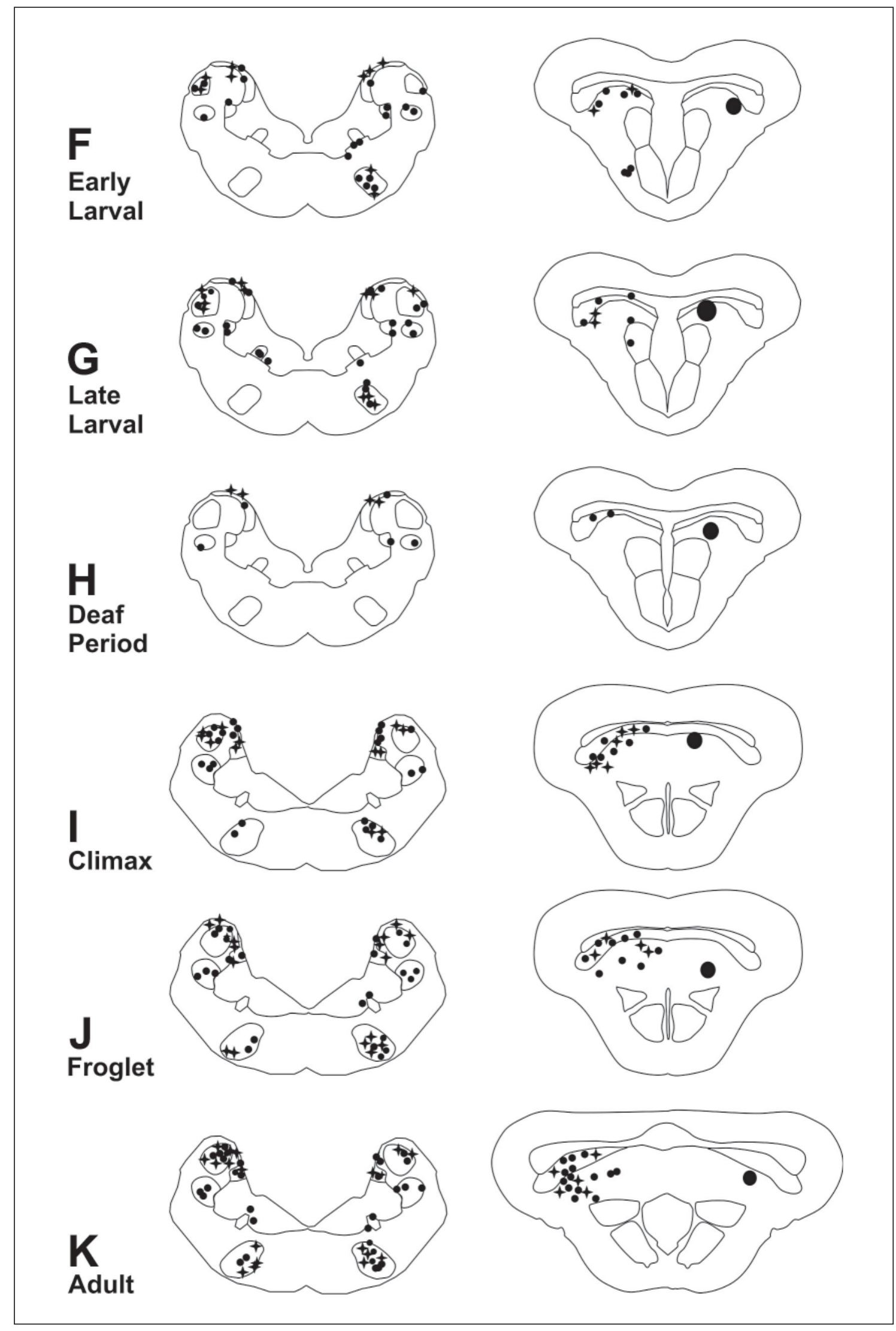

Metamorphic Changes in Auditory

Brain Behav Evol 2007;69:1-19 

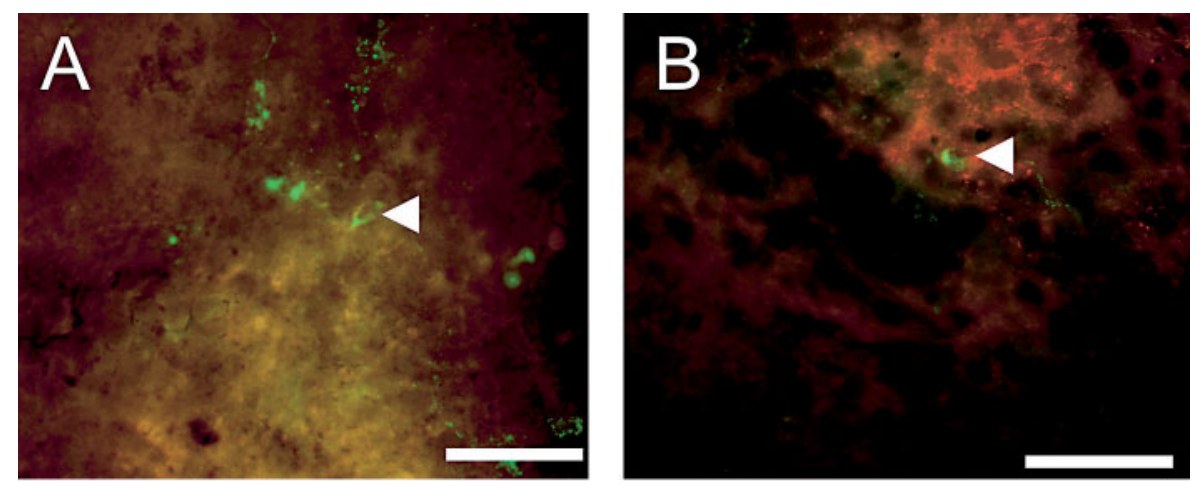

Fig. 3. CT $\beta$ filled cells (green) and PHA-L labeled terminals (red) following coinjection of CT $\beta$ and PHA-L into the SON of a stage 32 late larval tadpole $(\mathbf{A}, \mathbf{C}, \mathbf{E}, \mathbf{G})$ and a stage 40 deaf period tadpole (B, D, F, H). Data from the deaf period tadpole are derived from a large injection site filling the SON. A, B: Ipsilateral DMN. C: Contralateral LVN. D: Contralateral MVN. E, F: Contralateral SON. G, H: Ipsilateral TS. White arrows point to examples of labeled cell bodies. Some segments of CT $\beta$ labeled fibers are present in some panels. There is less label in the cSON and iTS of deaf period animals than in late larval animals. Scale bars $=50 \mu \mathrm{m}$.
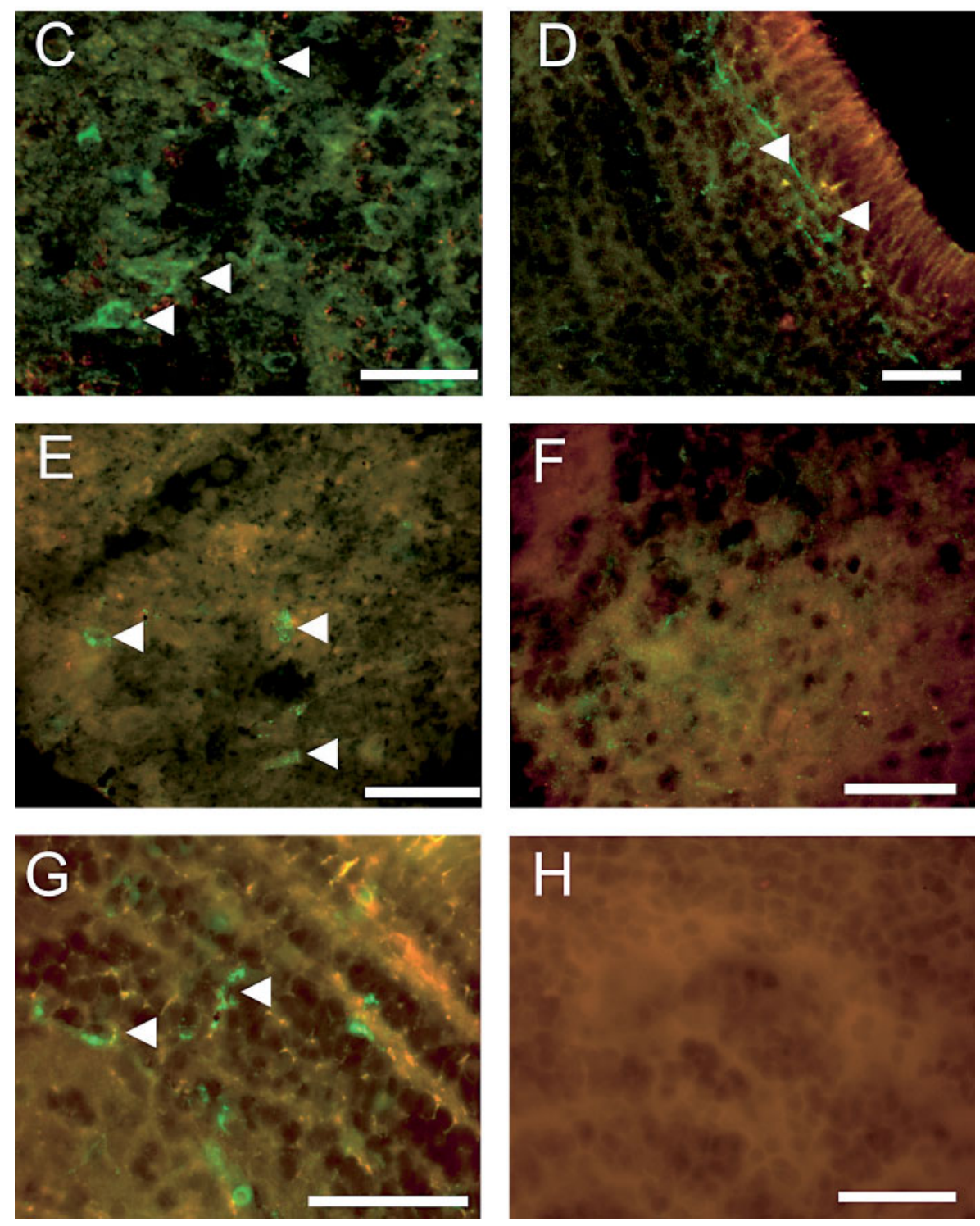

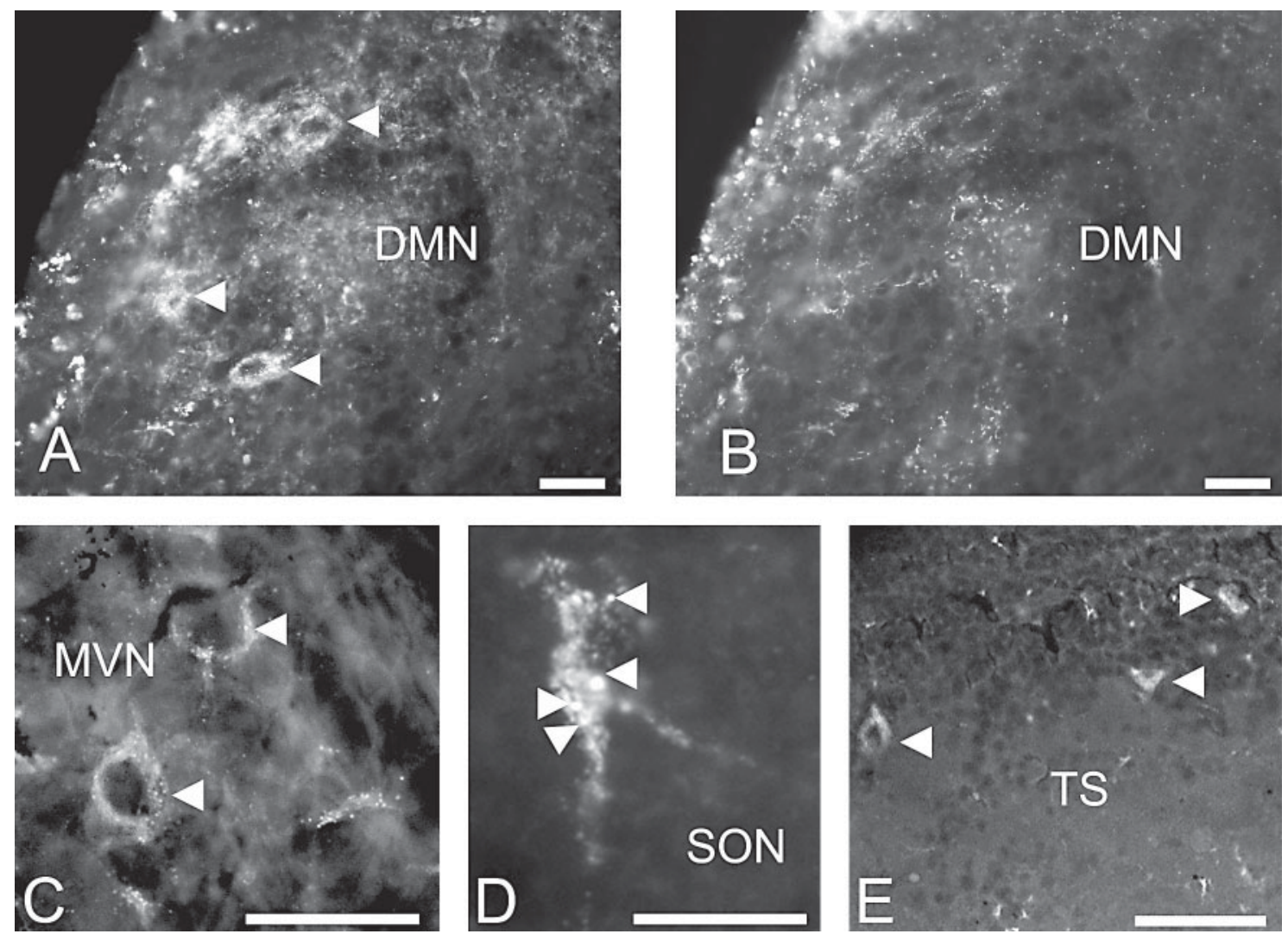

Fig. 4. High power shots of label in the medulla and midbrain of a stage 35 late larval tadpole following co-injection of PHA-L/ $\mathrm{CT} \beta$ into the TS. Images were converted to black and white from an RGB file. White arrows point to examples of labeled cell bodies. A CT $\beta$-labeled cells in the contralateral DMN derived from the green channel of the RGB file. Three cells are shown. B PHA-L labeled puncta/terminals derived from the red channel of the same file. These images show the presence of both labeled cell bodies and terminals in the cDMN. Label in the iDMN was sparser (see text). C CT $\beta$-labeled cells in the contralateral MVN. Two cells are shown. Label in the ipsilateral MVN was similar (see text). D CT $\beta$-labeled cell in the ipsilateral SON. This cell also showed PHA-L terminal label (bright perikaryal highlights). There was no cell or terminal label in the contralateral SON. E CT $\beta$-labeled cells in the contralateral TSl. Two cells are shown. Dorsal is up, medial is to the right in all images. Scale bars A-C: $50 \mu \mathrm{m}$; D, E: $25 \mu \mathrm{m}$. late larval stages, no terminal label was observed in the cTS.

Injections of DiI produced label in the immediate region of the SON and in fibers projecting dorsally towards the medial reticular zone (fig. 5A). Light to modest fiber label, depending on the size of the injection site, bilaterally overlaid the iDMN and the MVN. No labeled fibers were observed terminating in or near the $\mathrm{CDMN}$, and no fibers were observed projecting to the region of the TS even after large injections.

TS Injections. Injections of retrograde tracer into the TS (fig. 5B) yielded light cell body label in the cMVN and cTS, but no cell or fiber label in the iSON, even after large injections. There was sparse cell body label in the cDMN and iMVN and light cell body label in the LVN bilater- ally. Large injections produced sparse cell body label in the iDMN. Sparse cell body label in the ipsilateral LLa was observed in early deaf period (stages 38-39) animals. Only at the very end of the deaf period (morphologically between stages 41 and 42) did light fiber and sparse cell label begin reappearing in the iSON.

Anterograde label following PHA-L and DiI injections in the TS yielded light terminal label in the MVN bilaterally, and sparse to light terminal label of the DMN bilaterally and of the medial reticular region. Sparse terminal label was visible in the iSON but there was no terminal label visible in the cSON. DiI injections in the TS showed light fiber label in the cTS, modest fiber label in the ipsilateral lateral lemniscal tract, and light to modest label along the DAP and in the MVN bilaterally. As the deaf 

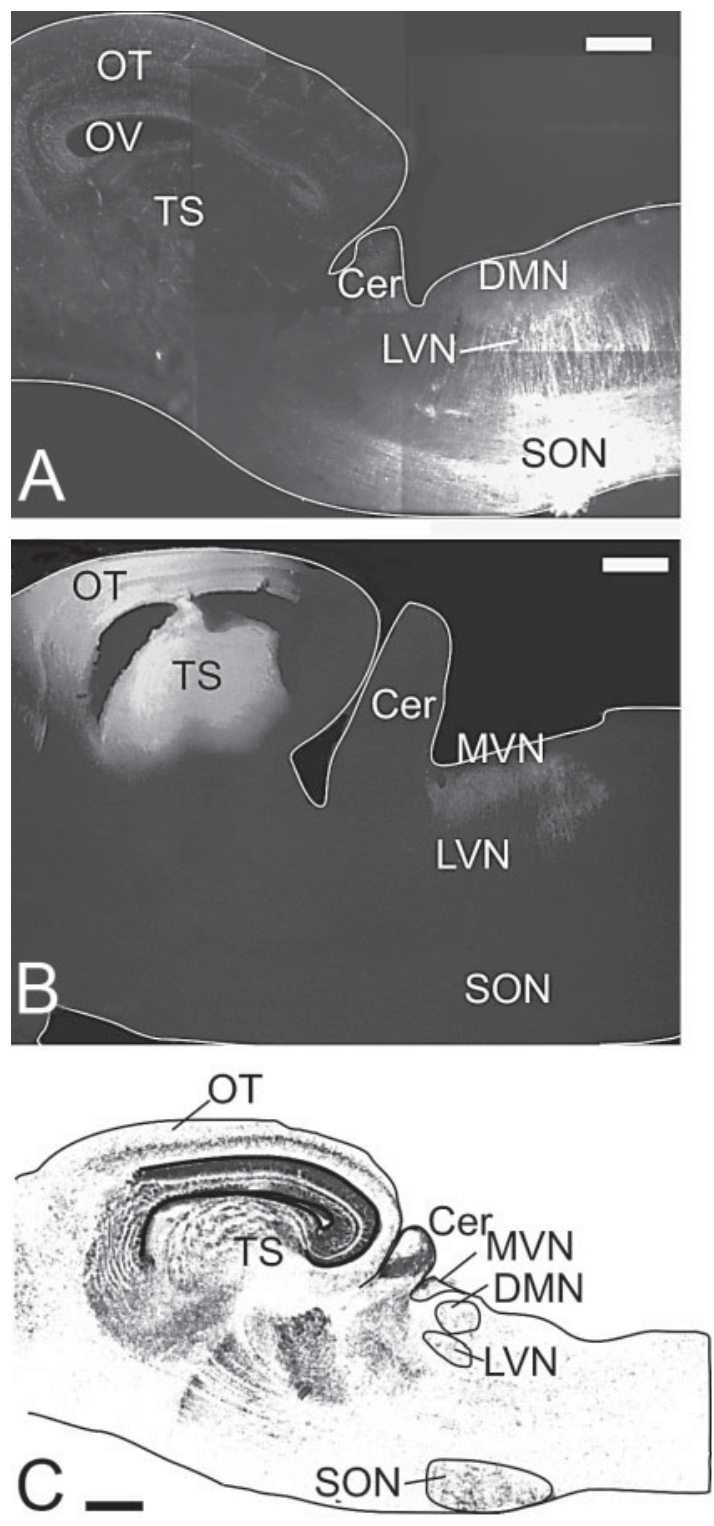

Fig. 5. Disruption of SON-TS connectivity in deaf period tadpoles. Images have been converted to grayscale from RGB files. A Sagittal section from a stage 40 tadpole, at a lateral (level of DMN) level. A large DiI injection into the SON shows fiber labeling in the dorsal medulla, extending up to the LVN and DMN. Even following a large tracer injection, there is only a limited spread of label rostrally. No travel to the TS itself can be observed. Internal edge lines are artifacts from mosaic combination of multiple low light level images. B Sagittal section from a more medial (level of MVN) level from a stage 41 tadpole. A large injection of DiI into the TS shows local propagation through the TS around the injection site and sparse label of the MVN region. Label in the optic tectum is likely due to leakage as the micropipette was lowered through this area. Even with large injection sites, there was no travel to the SON. Both sagittal sections have been heavily contrast enhanced to show any fiber label. Schematic of a sagittal section at the approximate level of $\mathbf{A}$ is shown in the bottom panel. Scale bars: $200 \mu \mathrm{m}$. period proceeded, fiber label overlying the DMN progressed from sparse to light.

\section{Metamorphic Climax Stages (42-46)}

Considerable differences in the pattern of connectivity were seen in the brainstem of tadpoles in metamorphic climax (fig. 2D, I), compared to those in all earlier larval stages. Although some of these changes reflect disconnection with the degenerating lateral line system, others show the reappearance of SON-iTS connectivity and the first emergence of SON-cTS connectivity.

SON Injections. Retrograde tracer injections into the SON yielded modest cell label in the cSON, cDMN, cLVN, and bilaterally in the MVN. Light label of cells in the iDMN, iLVN, and in the medial reticular region overlying the SON was present. No cell body label was observed in the region of the LLa, even in the youngest animals of this stage group when the nucleus was still present, although degenerating. Light to modest cell body label was observed in both the iTS and the cTS (fig. 6B, C). These labeled cells were scattered throughout the medial and lateral regions, compared to the more restricted distribution of labeled iTS cells seen in earlier stages. Sparse to light cell body label was also observed for the first time in deep layers of the lateral optic tectum.

Anterograde label after injection of PHA-L or DiI into the $\mathrm{SON}$ yielded modest terminal label in the $\mathrm{CDMN}$ and a progressive increase from light to modest label in the iDMN as the animals developed through climax. Modest label was also observed in the cSON, with light label in the MVN and LVN bilaterally. As with retrograde label, no anterograde label was observed in the LLa following onset of climax. Modest terminal label was observed in the iTS, particularly in lateral and ventral regions. Light label was present for the first time in the cTS, again mostly in the lateral regions. Injection of DiI into the SON resulted in moderate fiber label of the VAP, and modest fiber label in the cSON. The DAP showed light to modest bilateral fiber label extending to and terminating bilaterally in the DMN, LVN and the MVN. In addition, a clear fiber pathway is visible passing through the lateral lemniscal tract and extending into the TS and the deep layers of the optic tectum (fig. 6A).

TS Injections. Retrograde tracer injection in the TS yielded light to modest cell body label bilaterally in the DMN, LVN and MVN (fig. 6D, E), with the amount of label increasing as the animals progressed through climax. No cell body label was observed in the LLa. Light to modest cell body label was observed bilaterally in the SON (fig. 6F, G), as well as in the medial reticular zone. 
Fig. 6. Patterns of connectivity in metamorphic climax tadpoles. A Sagittal section through the brain of a stage 44 tadpole. DiI-Ph injection into the SON results in a clear fiber pathway (white arrows) extending into the TS and discrete fiber label of layers of the OT. Label of the Purkinje cell layer of the cerebellum may be due to uptake of dye by MVN cells that project both to the cerebellum and the SON. Scale bar: $200 \mu \mathrm{m}$. B-G Coronal sections through the medulla of a stage 44 tadpole after CT $\beta /$ PHA-L injection into the TS. White arrows indicate labeled cell bodies. B Contralateral TSl. C Ipsilateral TSl. D Contralateral DMN, MVN, LVN. Labeled cells are shown in all 3 nuclei. E Ipsilateral DMN, MVN, LVN. Labeled cells are shown in all 3 nuclei. $\mathbf{F}$ Contralateral SON. G Ipsilateral SON. B, D, F: Dorsal up, medial to the right. C, E, G: Dorsal up, medial to the left. Scale bars: $100 \mu \mathrm{m}$.
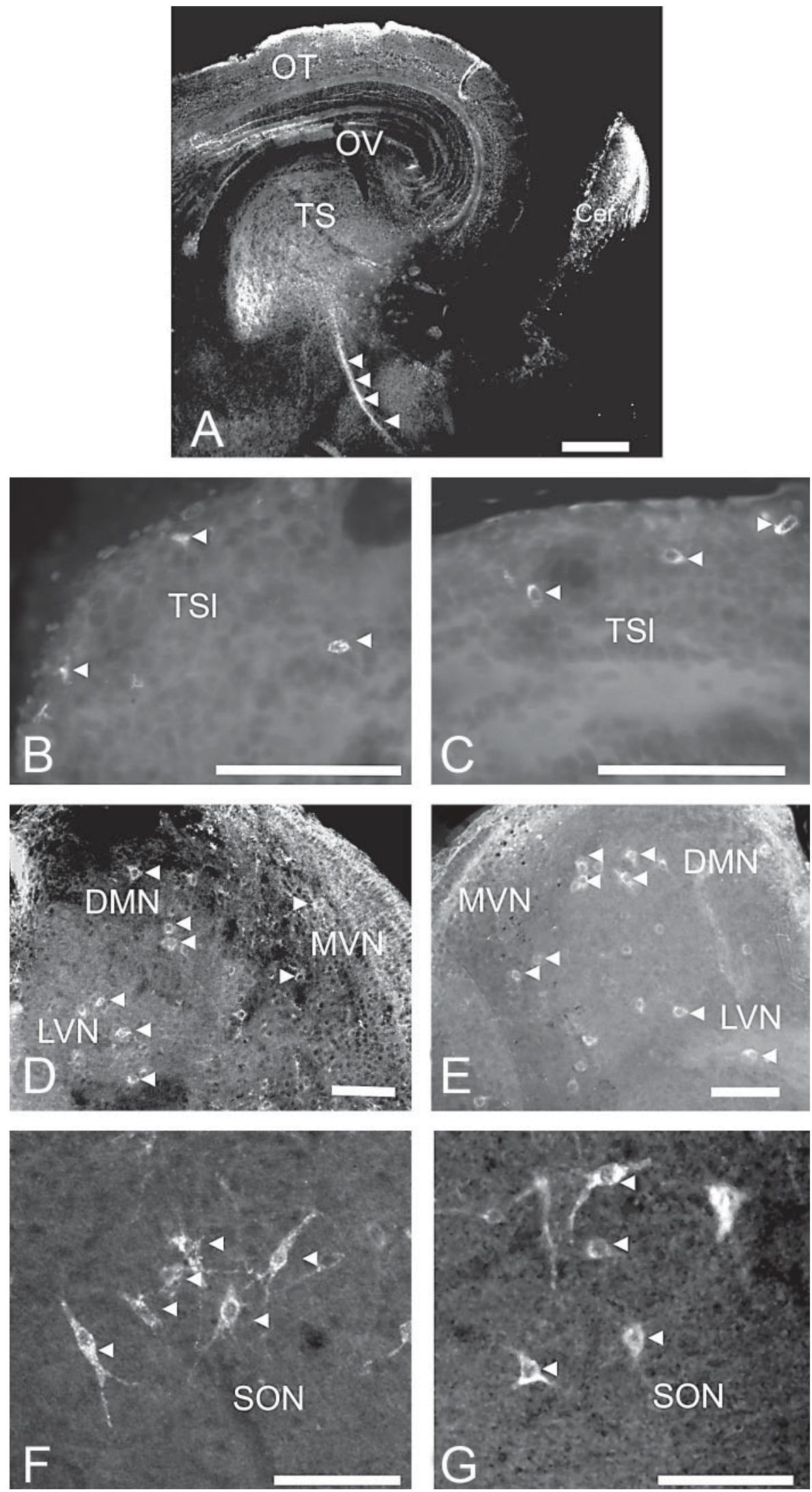
Moderate cell body label was seen in the cTS, particularly in the laminar and lateral regions.

Anterograde label following PHA-L or DiI injection in the TS yielded modest terminal label in the dorsal border of the $c D M N$ and in the mid and dorsal regions of the iSON. Light label was observed in the dorsal border of the iDMN, in the cSON, and bilaterally in the MVN. No terminal label was observed in the LVN. There was light to modest terminal label in the cTS, and sparse to light fiber label in the lateral lemniscal tract. DiI and HRP injections produced light fiber label overlying and terminating near the cDMN and bilaterally at the MVN. Sparse fiber label was observed near the iDMN. There was an increase in fiber labeling from the TS to the iSON via the ipsilateral lateral lemniscal tract during the climax developmental stages.

\section{Postmetamorphic Animals}

Our results in postmetamorphic animals are generally consistent with previous descriptions of auditory medullary/midbrain connectivity in adult Rana [Feng, 1986; Wilczynski, 1981; Feng and Lin, 1991].

SON Injections. Retrograde tracer injections into the $\mathrm{SON}$ of a froglet (fig. 2E) showed modest cell body label in the $\mathrm{cSON}$ and $\mathrm{CDMN}$, and bilaterally in the $\mathrm{MVN}$, $\mathrm{LVN}$ and throughout the medial reticular zone. Light but highly discrete bilateral label was also observed in some motor cells of the VII motor nucleus. Light cell body label was also observed in the iDMN. Light to modest label was present in the ipsilateral TSl, depending on the size of the injection, and in the area of ipsilateral principal nucleus. Sparse to light label was observed in several layers of the ipsilateral lateral optic tectum. Light to modest cell body label was also apparent in the cTS.

Anterograde label following PHA-L and DiI injections in the SON yielded modest terminal label in the $\mathrm{CSON}$, modest label in the DMN bilaterally, and light label of the MVN and LVN bilaterally. Light to modest terminal label was observed bilaterally in the TS. DiI injections in the SON showed patterns very similar to those observed in metamorphic climax, although heavier fiber label was observed along the DAP, and fewer labeled fibers were observed overlying the ipsilateral MVN than previously observed in tadpoles.

TS Injections. Focal injections of retrograde tracer into the froglet TS resulted in modest cell body label of the iSON (fig. 7A), iMVN (fig. 7B), and $\mathrm{cDMN}$ (fig. 7C). There was modest label of the LVN bilaterally. Light cell body label was observed in the cSON, iDMN (fig. 7B) and cMVN (fig. 7C). These injections also yielded moderate
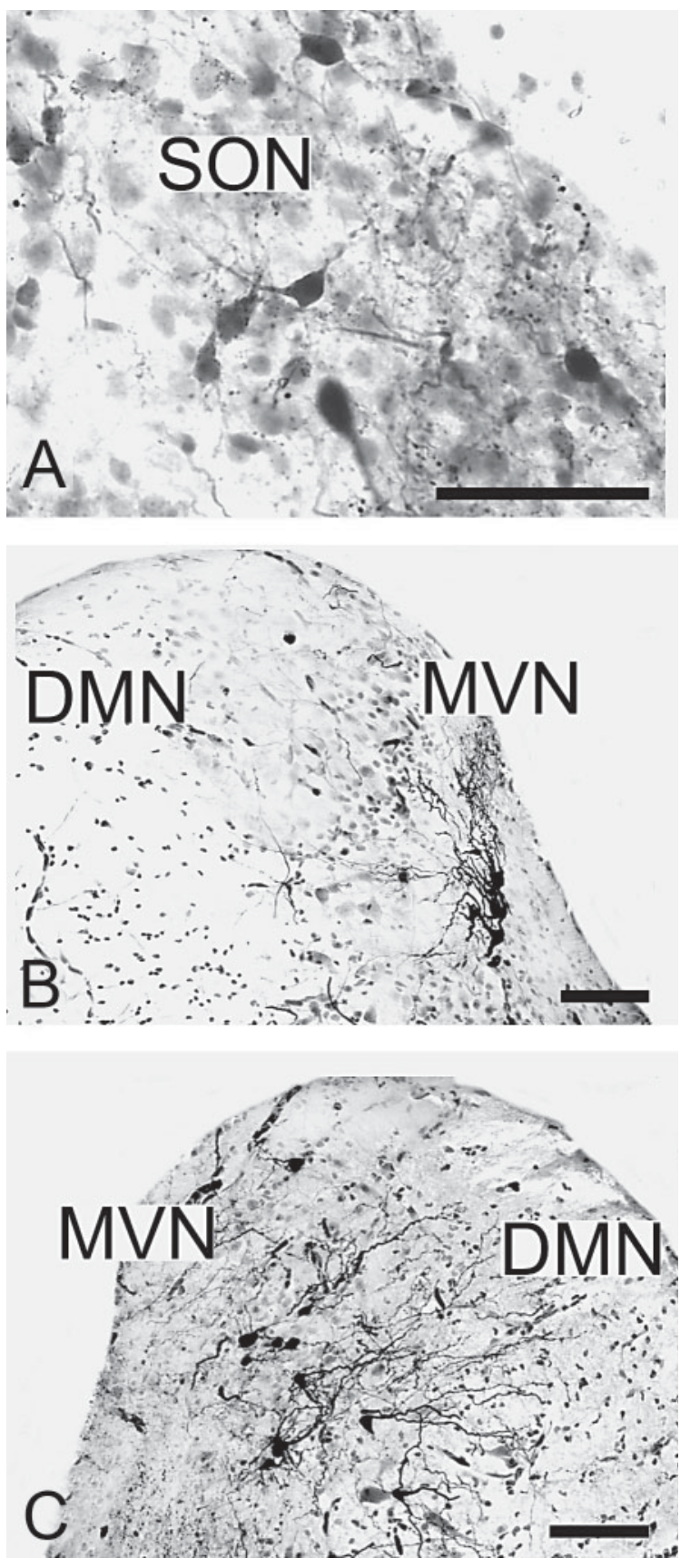

Fig. 7. Retrograde label in the medulla of a froglet (26 day postmetamorphosis) following HRP injections into the TS. Labeled cells and fibers are black. A Modest cell body label in the ipsilateral SON. B Ipsilateral dorsal medulla showing modest cell body and moderate fiber label in the MVN, and light label of DMN cells. C Contralateral dorsal medulla showing modest label of DMN cells and moderate label of fibers. There is only light label of the MVN region in this section. A, B: dorsal up, medial to the right. C: dorsal up, medial to the left. Scale bars: $100 \mu \mathrm{m}$. 


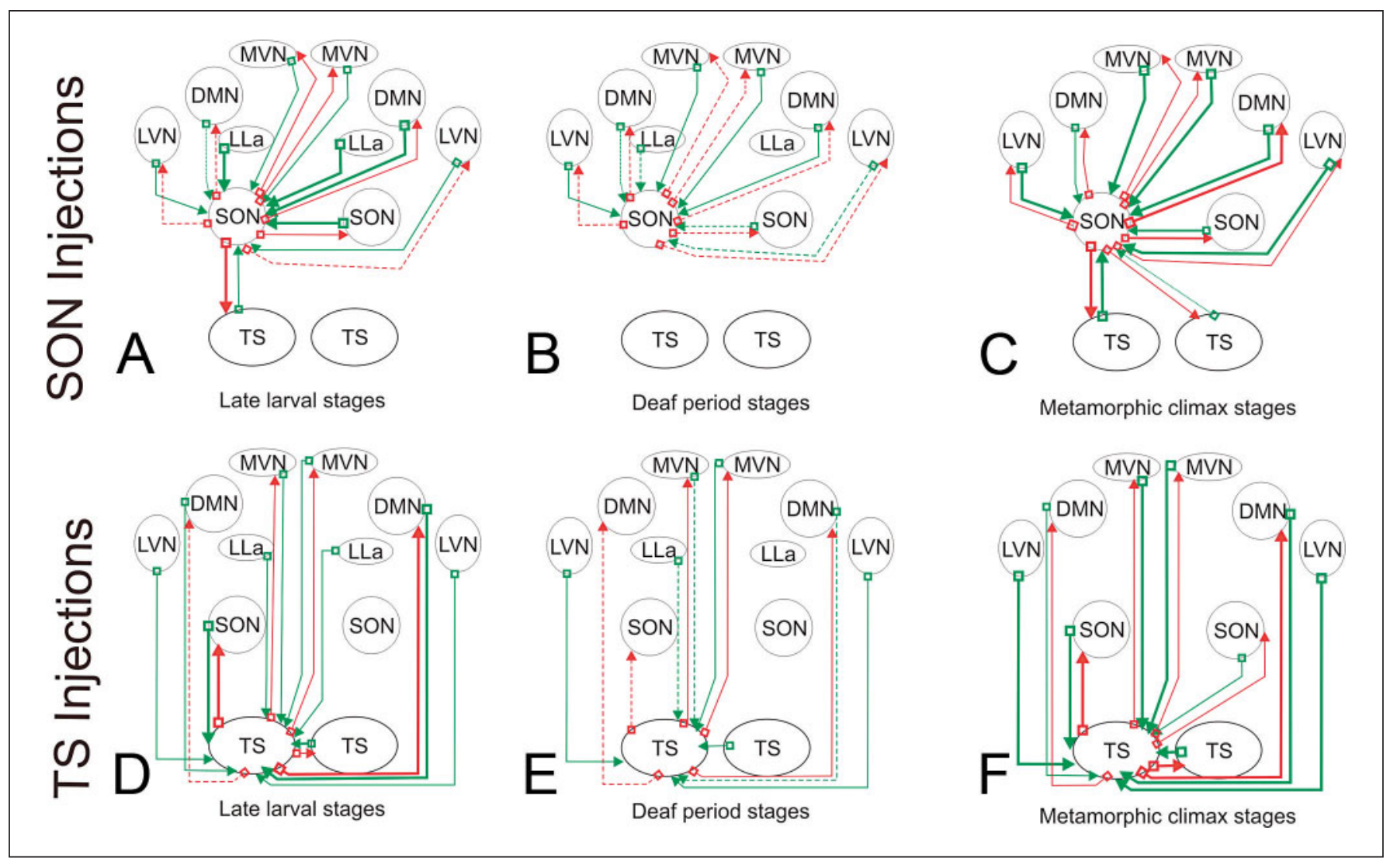

Fig. 8. Schematic of connectivity changes following SON injections (A-C) and TS injections (D-F) across 3 stages of metamorphic development. Arrows indicate direction of connectivity and line weight (heavy, light, dotted) indicates relative number of terminals and/or cell bodies based on anterograde (red) and retrograde (green) tracers.

cell body label in the cTS, widely distributed in and around the TSl as well as in the area of the principal nucleus (fig 2J). Adult frogs (fig. $2 \mathrm{~K}$ ) showed similar patterns, but with an increase in numbers of labeled cell bodies, particularly in the iSON and cTS.

PHA-L injections produced modest terminal label of the $\mathrm{CDMN}$ and iSON, light terminal label of the iDMN, and light terminal label in the MVN bilaterally. Consistent with the results in larval animals, no terminal label of the LVN was observed. Regardless of TS injection site, light to modest terminal label of the cTS, particularly the TSl, was seen. The pattern of fiber label after HRP and DiI injections was consistent between froglets and adults, although label was qualitatively greater in adults. Fiber label was seen, to differing extents, bilaterally in the DMN, SON, MVN and LVN, as well as in the cTS. In froglets, the greatest amount of fiber label was observed in the iMVN, cDMN and cTS, whereas in adults the greatest amount of label was observed in the iSON, iMVN, cDMN and cTS.

\section{Discussion}

Using multiple tracers and injection sites, we obtained a consistent pattern of results that both confirm and expand upon previous descriptions of central auditory neuroanatomy in larval Rana [Jacoby and Rubinson, 1984; Boatright-Horowitz and Simmons, 1997]. A summary of our data showing the pattern of developmental anatomical change between the late larval period and metamorphic climax is shown in figure 8 . These data reveal considerable reorganization of auditory pathways across metamorphic development, beyond those previously described. Our new findings include (1) afferent connectivity between the SON and the LVN emerges during late larval stages, and persists until the froglet period; (2) afferent connectivity between the SON and iDMN first emerges during late larval stages; (3) efferent connectivity between the SON and the iTS is disrupted during the deaf period; (4) afferent and efferent connectivity be- 
tween the SON and cTS first emerges during metamorphic climax; and (5) more extensive distribution of retrograde cell body label in the iTS after SON tracer deposits is observed beginning in climax. In addition, we show the stability of other pathways across development, including (1) afferent and efferent connectivity between the SON and MVN bilaterally, (2) afferent and efferent connectivity between the SON and cDMN, (3) afferent and efferent connectivity between the TS and cDMN, and (4) afferent connectivity between the cTS and iTS.

\section{Technical Considerations}

Technical limitations that may constrain interpretation of these data include the microinjection of a criterion amount of tracer into all brains, regardless of developmental stage; variable extent of injection sites, even with deposit of a criterion amount of tracer; and difficulties in distinguishing between failure of transport and failure of tracer uptake in mediating patterns of label. We attempted to alleviate the first two limitations by adopting a consistent injection and processing technique for all developmental groups. We chose to inject a criterion amount of tracer so that the hypothesized decrease in travel during the deaf period could not be attributed to less dye being injected in this stage group. Injection sites could differ in size, even within the same developmental group; this is likely due to differences in spread of tracer. We noted in the text variability in results that could be attributed to this variable. We emphasize, however, that the failure of transport to the TS in the deaf period (fig. 5A) is seen even after large injections into the SON. Other transport patterns, particularly within the medulla, could be subject to false positive results due to transneuronal labeling [Fritzsch et al., 1984; Birinyi et al., 2001] or to label of fibers of passage [Cliffer and Giesler, 1988]. For example, we observed, in late larval through metamorphic climax stages, label of VII motor nucleus cells following large injections of CT $\beta$ into the SON. It is possible that this cell body label does not represent a direct connection between the SON and VII motor nucleus, but is a result of uptake of tracer by the extensive dendritic branches of the VII motor neurons extending into the area of the DAP. Our use of a dorsal injection approach in some CT $\beta$ injections and the large size of cells and processes in VII motor nucleus prevent eliminating this hypothesis, although it is unclear why this label would appear in only certain developmental stages and not others. The presence of label in the MVN and LVN after SON injections could be a result of tracer uptake by the vestibular commissural fibers passing close to the injection site [Gro- fova and Corvaja, 1972]. This interpretation does not easily account for the observations that LVN label does not appear until late larval stages and that LVN label is absent in adults [Feng, 1986].

Our data do not definitively distinguish between failure of transport and failure of uptake by either cells or fibers. The observation that, during the deaf period, connectivity of the SON with the DMN, MVN and LVN persists at the same qualitative level as seen in late larval animals shows that medullary-medullary transport still occurs within this developmental period. We also observe cell body label in the iTS after SON injections in both early and late larval animals, suggesting that the lack of label in deaf period animals is due to some specific failure within that unique developmental time. Failure of uptake could occur in cells around the injection site, or in cells/terminals at the target site. It could also reflect differences in fiber- and cell-packing density either around the injection site or in the target nucleus, or in factors such as increased cell death or axonal degeneration within or around specific nuclei at particular stages of development. Cell number increases in auditory brainstem nuclei across development, with specific waves of new cell proliferation occurring during certain stages [Chapman et al., 2006; Simmons et al., 2006]. Variability in transport patterns may be related to these stage-specific changes in numbers of post-mitotic cells. We observed a qualitatively greater distribution of label, particularly in the TS, in metamorphic climax and froglet animals compared to that in other larval stages. This suggests that younger tissue may simply not label or process as well as more mature tissue, perhaps due to differences in extent of dendritic arborizations. At least in the SON, soma size does not consistently increase across larval development [Templin and Simmons, 2005].

\section{Reorganization of Medullary Connectivity across \\ Development}

In adult $R$. pipiens, HRP injections into the SON retrogradely label cell bodies in the cSON and in the DMN and MVN bilaterally, but do not label cell bodies in the LVN [Feng, 1986]. These results are generally consistent with what we observed based on the retrograde transport of CT $\beta$ and DiI/DiI-Ph in larval bullfrogs, but with three exceptions. One exception is the decline of label in the cSON specifically during the deaf period. This suggests either a failure of transport in the commissural pathway leading to the cSON, or a failure of uptake in target cells. A second exception is the absence of cell body label in the iDMN in early larval stages, which first emerges but re- 
mains sparse during late larval and deaf period stages, prior to increases during metamorphic climax. This result suggests a slower maturation of the ipsilateral, compared to the contralateral, DMN-SON pathway.

The third exception involves cell body label of the LVN after SON injections, which first appears in late larval stages and persists in froglets. We also observed anterograde terminal label in the LVN in this same developmental span. This result suggests reciprocal connectivity between the LVN and the SON in larval stages. Because such a pattern has not been reported in adult frogs [Feng, 1986], it could be a transient phenomenon, of biological relevance to the animal only during larval life. In adult frogs, the LVN, through its connections with the lateral vestibulospinal tract, plays an important role in equilibrium and the control and stabilization of postural responses. With the appearance of forelimbs during metamorphic climax, changes in postural stability emerge. By the completion of climax, postural reflexes shift from swimming and maintaining position while in the water column to those that facilitate walking and hopping on a solid substrate. In larval frogs, SON-LVN connectivity might facilitate the initial development and maturation of orienting behavior towards sound sources in air, a behavior which would emerge as the animal prepares to become amphibious and begins to adopt additional body positions (that is, not swimming or floating). Experiments utilizing direct tracer deposits into the LVN of larval animals are needed to confirm these results.

The presence during larval development of labeled cell bodies in the MVN after retrograde tracer injection into the SON suggest an important biological function of the MVN-SON pathway. One possibility is that auditory responsiveness in tadpoles is mediated by connections of the MVN to both the SON and the TS. This pathway could explain the residual auditory responsiveness of the TS sometimes seen during the deaf period [BoatrightHorowitz and Simmons, 1997]. Experiments utilizing direct tracer deposits into the MVN are needed to confirm these results as well.

\section{Medullary/Midbrain Connectivity across \\ Development}

Consistent with data in adult frogs [Pettigrew, 1981; Wilczynski, 1981; Feng and Lin, 1991], in all larval stages, retrograde tracer injections into the TS results in labeled cell bodies in the cTS. This commissural pathway is thus a stable feature of TS connectivity across development.

Our data also show organizational changes in connectivity between the TS and medullary nuclei mediating auditory, vestibular and lateral line modalities. Label of the CTS after tracer deposits in the SON first emerges during metamorphic climax stages, and remains in both froglets (this study) and adult frogs [Feng, 1986]. Jacoby and Rubinson [1984] injected HRP 'in the vicinity of' the SON of stage 35-37 bullfrog tadpoles (within our late larval stage), and observed cell body label in the medial/ventral region of the iTS. They did not report label in the cTS. The presence of label in the iTS is consistent with what we report here, although we observed label primarily in the lateral area of the TSl rather than the medial/ventral TS. The difference in location of cell body label between these two studies could be due to differences in the size of injection sites, the different kinds of tracers used, or to differences in terminology used to define areas of the TS.

In adult anurans, retrograde label of the iSON is consistently seen after HRP deposits in the TS [Pettigrew, 1981; Wilczynski, 1981; Feng and Lin, 1991]. Similarly, retrograde label of the iSON after HRP deposits into the TS is observed in some larval stages [Jacoby and Rubinson, 1984; Boatright-Horowitz and Simmons, 1997], except for the deaf period [Boatright-Horowitz and Simmons, 1997]. We now show that the changes in SON-TS connectivity are reciprocal. An additional feature of brainstem organization revealed in the current study is the presence of both retrograde and anterograde labeled cells in the iSON after TS injections. This type of label was seen only in early and late larval groups. It suggests the existence of a modulatory pathway between the TS and the iSON within these developmental times, with TS efferents providing putative feedback to afferent SON sources. The function of this pathway is unknown.

We observed the presence of afferent connectivity between the MVN bilaterally and the TS at all stages of development. Similarly, both Wilczynski [1981] in adult Rana and Pettigrew [1981] in adult Bufo observed the presence of afferents from the caudal nucleus bilaterally to the TS. Feng and Lin [1991] did not explicitly discuss these connections, but from their figures it appears that there are retrogradely labeled cells in the MVN after HRP deposits in the TS. We also note connectivity between the LVN and the TS at all stages of development, consistent with results in adults [Pettigrew, 1981; Wilczynski, 1981; but see Matesz et al., 2002]. We also show, in early and late larval stages, the presence of spase to light retrogradely labeled cells bilaterally in the LLa after TS injections. Boatright-Horowitz and Simmons [1997] reported 'considerable' cell label in the LLa during these stages after injections into the lateral TS. The label begins to dis- 
appear during the deaf period, and is totally absent during metamorphic climax. This is consistent with previous reports [Jacoby and Rubinson, 1983; Fritzsch et al., 1984] of the degeneration of lateral line afferents and nuclei in non-pipid frogs during metamorphic climax.

\section{Possible Mechanisms of Anatomical Reorganization}

As described in other developing systems, plasticity of central anatomical connections could be triggered by changes in peripheral sensory input [LeVay et al., 1980; Reh and Constantine-Paton, 1985; Galli and Maffei, 1988; Johnson and Bottjer, 1994]. Peripheral changes may analogously drive the anatomical reorganization of the auditory pathways during metamorphic development, particularly during the deaf period. In larval frogs, peripheral transduction pathways available for mediating sensory input from the otic capsule develop and mature at different time points (reviewed in Simmons and Horowitz, 2006]. One explicit change occurs with the development, during the deaf period, of the opercularis transduction pathway. This pathway (consisting of the operculum and the opercularis muscle/shoulder girdle connection) replaces auditory sensation mediated by direct kinetic effects on otolithic organs (the fenestral pathway) which operates during early and late larval period [Hetherington, 1987]. The initial development of the opercularis pathway features a transient blockade of the oval window by the developing operculum cartilage, which would then diminish input to the inner ear. The loss of activity in the TS during the deaf period might reflect the absence of peripheral input to drive both functional activity and the maintenance of anatomical connectivity. The reemergence of activity in the TS during metamorphic climax could reflect renewed input to the inner ear, resulting from a partial retraction of the operculum as the stapes develops [Hetherington, 1987]. Moreover, during metamorphic climax, the tympanic/columellar transduction pathway begins to develop, maturing during the early froglet period when the external tympanum first appears on the side of the animal's head [Boatright-Horowitz and Simmons, 1995]. Further, central anatomical changes occurring during metamorphic climax and the froglet period would thus reflect both, the initial development and final maturation of this second transduction pathway, and the attendant increase in activity from the periphery that it entails. What this scenario does not answer is why the SON-TS pathway is specifically affected during the deaf period while the DMN-TS pathway remains relatively intact. This is an interesting question for future research.
The reorganization of central auditory pathways reported here presumably requires (or reflects) cell turnover, produced by waves of either cell proliferation or cell death. Chapman et al. [2006] observed stage-specific changes in cell proliferation in both the DMN and the SON, with peaks of cell birth in the early (DMN, SON) or late (DMN) larval periods, a decline during the deaf period, and a subsequent peak in metamorphic climax. Similarly, Templin and Simmons [2005] observed a decline in the number of differentiated neurons in the SON beginning during the deaf period and extending into the early stages of metamorphic climax. Numbers of neurons then increase during the later stages of metamorphic climax and the froglet period. A different pattern emerges in the TS, however. In this auditory nucleus, cell proliferation is low from hatchling to late larval stages, then increases during the deaf period to reach a peak at metamorphic climax [Simmons et al., 2006]. The peaks in cell proliferation in all three auditory brainstem nuclei at metamorphic climax might be related to the development of the tympanic/columellar pathway at this time period, but the variability in cell proliferation at earlier stages seems to reflect a different process that may be independent of changes in peripheral input.

One central mechanism that may mediate these effects independent of peripheral activation is changes in the levels of thyroid hormone and its receptors. Thyroid hormone levels drive many of the external morphological transformations appearing across metamorphosis [Shi, 2000]. In addition, changes in expression of its receptors are correlated with the remodeling of the retina and spinal cord of Xenopus larva over metamorphosis [MarshArmstrong et al., 1999, 2004]. How changes in the expression of thyroid hormone receptors might be involved in the reorganization of auditory pathways over metamorphosis is a subject for further research.

\section{Acknowledgements}

This research was supported by NIH grants R01 DC05257 (AMS, SSH), F31 DC06776 (JAC), and by the Rhode Island Space Grant Consortium. 


\section{References}

-Antal M, Petko M (1990) Retrograde transport of the lectin Phaseolus vulgaris Leucoagglutin in frog central nervous system. J Histochem Cytochem 38:1913-1917.

- Birinyi A, Straka H, Matesz C, Dieringer N (2001) Location of dye-coupled second order and of efferent vestibular neurons labeled from individual semicircular canal or otolith organs in the frog. Brain Res 921:44-59.

-Boatright-Horowitz SS, Simmons AM (1995) Postmetamorphic changes in auditory sensitivity of the bullfrog midbrain. J Comp Physiol A 177:577-590.

-Boatright-Horowitz SS, Simmons AM (1997) Transient 'deafness' accompanies auditory development during metamorphosis from tadpole to frog. Proc Natl Acad Sci USA 94: 14877-14882.

Boatright-Horowitz SS, Garabedian CE, Odabashian KH, Simmons AM (1999) Coding of amplitude modulation in the auditory midbrain of the bullfrog across metamorphosis. J Comp Physiol A 184:219-231.

-Chapman JA, Weinstein JL, Simmons AM (2006) Cell proliferation in the Rana catesbeiana auditory medulla over metamorphic development. J Neurobiol 66:115-133.

- Cliffer KD, Giesler GJ Jr (1988) PHA-L can be transported anterogradely through fibers of passage. Brain Res 458:185-191.

-Feng AS (1986) Afferent and efferent innervation patterns of the superior olivary nucleus of the leopard frog. Brain Res 364:167-171.

-Feng AS, Lin W (1991) Differential innervation patterns of three divisions of frog auditory midbrain (torus semicircularis). J Comp Neurol 306:613-630.

-Fritzsch B, Nikundiwe AM, Will U (1984) Projection patterns of lateral line afferents in anurans: A comparative HRP study. J Comp Neurol 229:451-469.

Fritzsch B, Wahnschaffe U, Bartsch U (1988) Metamorphic changes in the octavolateralis system of amphibians. In: The Evolution of the Amphibian Auditory System (Fritzsch B, Ryan MJ, Wilczynski W, Hetherington TE, Walkowiak W, eds), pp 359-376. New York: Wiley.

-Galli L, Maffei L (1988) Spontaneous impulse activity of rat retinal ganglion cells in prenatal life. Science 242:90-91.

-Gerfen CR, Sawchenko PE (1984) An anterograde neuroanatomical tracing method that shows the detailed morphology of neurons, their axons and terminals: immunohistochemical localization of an axonally transported plant lectin, Phaseolus vulgaris leucoagglutinin (PHA-L). Brain Res 290: 219-238.

-Godement P, Vanselow J, Thanos S, Bonhoeffer F (1987) A study in developing visual systems with a new method of staining neurones and their processes in fixed tissue. Development 101:697-713.
Gosner KL (1960) A simplified table for staging anuran embryos and larvae with notes on identification. Herpetologica 16:183-190.

Grofova I, Corvaja N (1972) Commissural projection from the nuclei of termination of the VIIIth cranial nerve in the toad. Brain Res 42:189-195.

Hanker JS, Yates PE, Metz CB, Rustioni A (1977) A new specific sensitive and non-carcinogenic reagent for the demonstration of horseradish peroxidase. Histochem J 9:789-792.

Hetherington TE (1987) Timing of development of the middle ear of Anura. Zoomorphology 106:289-300.

Jacoby J, Rubinson K (1983) The acoustic and lateral line nuclei are distinct in the premetamorphic frog, Rana catesbeiana. J Comp Neurol 216:152-161.

Jacoby J, Rubinson K (1984) Efferent projections of the torus semicircularis to the medulla of the tadpole, Rana catesbeiana. Brain Res 292:278-381.

Johnson F, Bottjer SW (1994) Afferent influences on cell death and birth during development of a cortical nucleus necessary for learned vocal behavior in zebra finches. Development 120:13-24.

Kumaresan V, Kang C, Simmons AM (1998) Development and differentiation of the anuran auditory brainstem across metamorphosis: An acetylcholinesterase histochemical study. Brain Behav Evol 52:111-125.

Larsell O (1934) The differentiation of the peripheral and central acoustic apparatus in the frog. J Comp Neurol 60:473-525.

LeVay S, Wiesel TN, Hubel DH (1980) The development of ocular dominance columns in normal and visually deprived monkeys. J Comp Neurol 191:1-51.

Luppi PH, Fort P, Jouvet M (1990) Iontophoretic application of unconjugated cholera toxin $\mathrm{B}$ subunit $(\mathrm{CTb})$ combined with immunohistochemistry of neurochemical substances: a method for transmitter identification of retrogradely labeled neurons. Brain Res 534: 209-224.

-Marsh-Armstrong N, Huang H, Remo BF, Liu TT, Brown DD (1999) Asymmetric growth and development of the Xenopus laevis retina during metamorphosis is controlled by type III deiodinase. Neuron 24:871-878.

Marsh-Armstrong N, Cai L, Brown DD (2004) Thyroid hormone controls the development of connections between the spinal cord and limbs during Xenopus laevis metamorphosis. Proc Natl Acad Sci USA 101:165-170.

Matesz C (1979) Central projection of the VIIIth cranial nerve in the frog. Neurosci 4:20612071.

Matesz C, Kulik A, Bacskai T (2002) Ascending and descending projections of the lateral vestibular nucleus in the frog Rana esculenta. J Comp Neurol 444:115-128.
McCormick CA (1999) Anatomy of the central auditory pathways of fish and amphibians. In: Comparative Hearing: Fish and Amphibians (Fay RR, Popper AN, eds), pp 155-217. Springer: New York.

McDiarmid RW, Altig R (1999) Research: Materials and techniques. In Tadpoles: The Biology of Anuran Larvae (McDiarmid RW, Altig R, eds), pp 7-23. Chicago IL: University of Chicago Press.

Montgomery N (1988) Projections of the vestibular and cerebellar nuclei in Rana pipiens. Brain Behav Evol 31:82-95.

-Opdam P, Kemali M, Nieuwenhuys R (1976) Topological analysis of the brainstem of the frogs Rana esculenta and Rana catesbeiana. J Comp Neurol 165:307-332.

- Pettigrew AG (1981) Brainstem afferents to the torus semicircularis of the Queensland cane toad. J Comp Neurol 202:59-68.

Reh TA, Constantine-Paton, M (1985) Eye-specific segregation requires neural activity in three-eyed Rana pipiens. J Neurosci 5:11321143.

- Reichenberger I, Straka H, Ottersen OP, Streit P, Gerrits, NM, Dieringer N (1997) Distribution of GABA, glycine, and glutamate immunoreactivities in the vestibular nuclear complex of the frog. J Comp Neurol 377: 149-164.

Shi YB (2000) Amphibian Metamorphosis: From Morphology to Molecular Biology. New York: Wiley.

-Simmons AM, Chapman JA (2002) Metamorphic changes in GABA immunoreactivity in the brainstem of the bullfrog, Rana catesbeiana. Brain Behav Evol 60:189-206.

Simmons AM, Horowitz SS (2006) Plasticity in the auditory system across metamorphosis. In: Hearing and Sound Communication in Amphibians (Narins PM, Feng AS, Fay RR, Popper AN, eds) In press. Berlin: SpringerVerlag.

Simmons AM, Chapman JA, Brown RA (2006) Developmental changes in cell proliferation in the auditory midbrain of the bullfrog, Rana catesbeiana. J Neurobiol, in press.

Spaeti U (1978) Development of sensory systems in the larval and metamorphosing European grass frog. J Hirnforsch 19:543-575.

Templin T, Simmons AM (2005) Cellular and spatial changes in the anuran superior olive across metamorphosis. Hear Res 207:87-98.

-Wilczynski W (1981) Afferents to the midbrain auditory center in the bullfrog, Rana catesbeiana. J Comp Neurol 198:421-433.

Will U, Fritzsch B (1988) The eighth nerve of amphibians: Peripheral and central distribution. In: The Evolution of the Amphibian Auditory System (Fritzsch B, Ryan MJ, Wilczynski W, Hetherington TE, Walkowiak W, eds), pp 159-183. New York: Wiley. 